\title{
Higher Quantum Geometry and Non-Geometric String Theory
}

\section{Richard J. Szabo*}

Department of Mathematics, Heriot-Watt University, Edinburgh, United Kingdom.

Maxwell Institute for Mathematical Sciences, Edinburgh, United Kingdom.

The Higgs Centre for Theoretical Physics, Edinburgh, United Kingdom.

E-mail: R.J.Szabo@hw.ac.uk

We present a concise overview of the physical and mathematical structures underpinning the appearence of nonassociative deformations of geometry in non-geometric string theory. Starting from a quick recap of the appearence of noncommutative product and commutator deformations of geometry in open string theory with $B$-fields, we argue on physical principles that closed strings should instead probe triproduct and tribracket deformations in backgrounds of locally non-geometric fluxes. After describing the toy model of electric charges moving in fields of smooth distributions of magnetic charge as a physical introduction to the notions of nonassociative geometry, we review the description of non-geometric fluxes in generalized geometry and double field theory, and the worldsheet calculations suggesting the appearence of nonassociative deformations, together with their caveats. We discuss how algebroids and their associated AKSZ sigma-models give a description of non-geometric backgrounds in terms of higher geometry, and consider the quantization of the membrane sigma-model which geometrizes closed strings with $R$-flux. From this we derive an explicit nonassociative star product for the quantum geometry of the closed string phase space, and apply it to derive the triproducts that appear in conformal field theory correlation functions, to describe a consistent treatment of nonassociative quantum mechanics, to demonstrate quantitatively the coarse-graining of spacetime due to $R$-flux, and to describe the quantization of Nambu brackets. We also briefly review how these constructions lead to a nonassociative theory of gravity, their uplifts to non-geometric M-theory, and the role played by $L_{\infty}$-algebras in these developments.

Proceedings of the Corfu Summer Institute 2017 "School and Workshops on Elementary Particle Physics and Gravity"

2-28 September 2017

Corfu, Greece

${ }^{*}$ Speaker. 


\section{Contents}

1. Introduction: String theory and higher noncommutative geometry 1

2. A first glimpse at nonassociative geometry: Magnetic monopoles 6

3. Non-geometric fluxes and nonassociative geometry 9

3.1 Generalized geometry 11

$\begin{array}{ll}3.2 & \text { Double field theory } \\ 3.3 & \text { Overview }\end{array}$

$\begin{array}{ll}3.3 \text { Overview of conformal field theory results } & 14\end{array}$

$\begin{array}{lll}3.4 \text { Caveats } & 16\end{array}$

4. Higher geometrization of non-geometry 17

$\begin{array}{lll}4.1 & \text { AKSZ theory } & 18\end{array}$

$\begin{array}{lll}4.2 & \text { Poisson sigma-models } & 19\end{array}$

$\begin{array}{lll}4.3 \text { Courant sigma-models } & 19\end{array}$

5. Quantization of non-geometric backgrounds 21

$\begin{array}{ll}5.1 \text { Quantization of topological string theory } & 21\end{array}$

$\begin{array}{lll}5.2 & \text { Triproducts } & 24\end{array}$

5.3 Nonassociative quantum mechanics 25

5.4 Spacetime quantization 28

5.5 Quantization of Nambu brackets 30

6. Further developments 31

$\begin{array}{lll}6.1 & \text { Nonassociative gravity } & 31\end{array}$

6.2 Higher structures in non-geometric M-theory 33

$6.3 L_{\infty}$-algebras 36

\section{Introduction: String theory and higher noncommutative geometry}

The second Training School of the COST Action Quantum Structure of Spacetime was devoted to the general topic "Quantum Spacetime and Physics Models". While this is a broad topic with many potential directions (some covered by other lectures at this School), for the purposes of these lectures this refers to the problem that Einstein's general theory of relativity cannot be consistently quantized via quantum field theory, due to the ultraviolet divergences that plague perturbation theory around a flat background which require infinitely-many counterterms. To attempt to solve this problem one can consider physics models with a natural minimal length providing a suitable ultraviolet regularization. In this contribution we consider two such theories and how to reconcile 
them within the context of the School. Our treatment in this section and some other portions of this article have been influenced by various other reviews available, such as e.g. [85, 94, 91, 26].

One approach is based on noncommutative geometry. In attempting to reconcile quantum mechanics with gravity, which is a theory based on the geometry of spacetime, one is inevitably led to the notion of 'quantum geometry', which refers to the application of the principles of quantum mechanics to spacetime itself. One way to think of such a quantization is by promoting the spacetime coordinates $x^{i}$ to 'operators' which do not commute:

$$
\left[x^{i}, x^{j}\right]=\mathrm{i} \hbar \theta^{i j},
$$

for some bivector $\theta^{i j}$ which naturally incorporates a minimal area: Applying the standard uncertainty principle to the commutation relations (1.1) implies $\Delta x^{i} \Delta x^{j} \geqslant \frac{\hbar}{2}\left|\theta^{i j}\right|$, so that the minimal value of $\left|\theta^{i j}\right|$ may be thought of as the Planck length $\ell_{\mathrm{P}}$ of spacetime. Quantum field theory on such noncommutative spaces exhibits very interesting features of forbidden interactions, controlled Lorentz violation, and UV/IR mixing (see e.g. [113] for a review), and it can be extended to a noncommutative theory of gravity $[14,13]$ as discussed in the lectures of L. Castellani at this School. However, there are two major pitfalls to this approach. Firstly, although a minimal length scale is naturally introduced, there is no coarse-graining of spacetime that one would expect from a quantum theory of gravity: An underlying discrete structure such as a quantum of minimal volume does not appear in this framework. Secondly, most treatments assume that the brackets (1.1) satisfy the Jacobi identity; in particular, when the bivector $\theta=B^{-1}$ is invertible, it is not clear how to deal with the cases with non-vanishing flux $H=\mathrm{d} B \neq 0$. As we will discuss in the following, these two drawbacks are in fact related and are simultaneously dealt with by extending noncommutative geometry into the world of nonassociative geometry, which deals with deformations by higher structures in geometry.

Another approach is based on string theory, which certainly provides a concrete physical model of a quantum spacetime. Strings are extended one-dimensional degrees of freedom and so, unlike the point particle probes of quantum field theory, naturally come with intrinsic minimal length $\Delta x^{i} \geqslant \ell_{s}$, where $\ell_{s}$ is the string length. The interactions of strings thus violate locality, while the theory directly contains gravity and is on-shell ultraviolet finite. It is then natural to ask whether the spacetime approaches based on noncommutative geometry and string theory are related or are complementary to each other in some sense. The precise connection between open strings and noncommutative geometry was discovered near the end of the last millenium, see e.g. $[52,8,9,103,45,104,101,102]$, and is by now well-established. Open strings on D-branes in background $B$-fields (which provide a gauge flux on the D-brane worldvolume) probe a noncommutative worldvolume geometry. The massless bosonic field content of open string theory consists of gauge fields $A_{i}$ on the worldvolume together with scalar fields $\phi^{a}$ governing the transverse fluctuations of the D-branes in spacetime. In the Seiberg-Witten scaling limit which decouples open and closed string modes, the effective low-energy dynamics is governed by a noncommutative gauge theory on the D-brane worldvolume.

Although this connection is precise, and has led to a flurry of investigation over the last 20 years, this does not explain the connection of noncommutative geometry with gravity, nor how noncommutative geometry can be used to formulate a consistent theory of quantum gravity. In the present context, one needs to look at closed strings. The massless bosonic field content of closed 
string theory consists of the spacetime metric $g_{i j}$, the Kalb-Ramond field $B_{i j}$, and the dilaton field $\Phi$. Thus the closed string sector contains the data of background geometry and gravity, and it is here that one should seek analogs of a quantum geometry and suitable decoupling limits to make contact with the problem of quantizing gravity. Such a connection should certainly appear if noncommutative geometry indeed improves the ultraviolet behaviour of quantum gravity.

The connections between noncommutative geometry and closed string theory has been a topic of increasing interest over the last eight years, where it has been realised that closed strings are related to not only noncommutative but even nonassociative target space geometries. To understand how nonassociativity can arise in closed string theory, it is helpful to take a step back and look at in more detail why noncommutativity emerges in open string theory. An instructive pedagogical analogy from quantum mechanics is provided by the Landau problem [111]: The planar quantum dynamics of electrons of mass $m$ and charge $e$ propagating under the influence of a perpendicularly applied constant background magnetic field of magnitude $B$. The Lagrangian is

$$
L=\frac{m}{2} \dot{\vec{x}}^{2}-e \dot{\vec{x}} \cdot \vec{A} \quad \text { with } \quad A_{i}=-\frac{B}{2} \varepsilon_{i j} x^{j} .
$$

The limit $B \gg m$ of strong magnetic field induces the projection onto the lowest Landau level, described by a first order Lagrangian $\left.L\right|_{m=0}=\frac{e B}{2} \dot{x}^{i} \varepsilon_{i j} x^{j}$ with degenerate phase space whose canonical quantization gives the commutation relations of a noncommutative space:

$$
\left[x^{i}, x^{j}\right]=\frac{\mathrm{i} \hbar}{e B} \varepsilon^{i j}=: \mathrm{i} \hbar \theta^{i j} .
$$

This simple model is analogous to that of bosonic open strings in a $B$-field background, which at tree-level in string perturbation theory is described generally by the worldsheet action

$$
S=\frac{1}{4 \pi \ell_{s}^{2}} \int_{\Sigma_{2}}\left(g_{i j}(x) \mathrm{d} x^{i} \wedge * \mathrm{~d} x^{j}-2 \pi \ell_{s}^{2} B_{i j}(x) \mathrm{d} x^{i} \wedge \mathrm{d} x^{j}\right)
$$

where $x^{i}$ are maps from the string worldsheet, which is a disk $\Sigma_{2}$, to the target spacetime $M$. The low-energy limit $\ell_{s} \rightarrow 0$ describes the decoupling of massive string states, while the Seiberg-Witten scaling limit $g_{i j} \sim \ell_{s}^{4} \rightarrow 0$ ensures that gravity is non-dynamical and that the bulk modes of the string decouple from the boundary degrees of freedom. In this limit the action (1.4) reduces to a simple topological action given by the pullback of the Kalb-Ramond two-form to the string worldsheet:

$$
\left.S\right|_{g, \ell_{s}=0}=-\frac{1}{2} \int_{\Sigma_{2}} B_{i j}(x) \mathrm{d} x^{i} \wedge \mathrm{d} x^{j}
$$

In the absence of NS-NS flux $H=\mathrm{d} B=0$, and for target space $M=\mathbb{R}^{d}$, using Stokes' theorem the action (1.5) gives a pure boundary interaction $\left.S\right|_{g, \ell_{s}=0}=-\oint_{\partial \Sigma_{2}} \mathrm{~d} t \overrightarrow{\dot{x}} \cdot \vec{A}$, where $B=\mathrm{d} A$ and $\dot{x}^{i}=\partial_{t} x^{i}$ denotes a tangential derivative of the string field along the worldsheet boundary circle $\partial \Sigma_{2}$. Again this action is of first order in worldsheet time derivatives, so that the open string endpoints have a degenerate phase space in the decoupling limit. For a constant $B$-field with the symmetric gauge choice $A_{i}=-\frac{1}{2} B_{i j} x^{j}$, the action becomes

$$
\left.S\right|_{g, \ell_{s}=0}=\frac{1}{2} \oint_{\partial \Sigma_{2}} \mathrm{~d} t x^{i} B_{i j} \dot{x}^{j}
$$


and if the $B$-field is moreover non-degenerate then its canonical quantization produces the noncommutative coordinate algebra

$$
\left[x^{i}, x^{j}\right]=\left(\frac{\mathrm{i} \hbar}{B}\right)^{i j}=: \mathrm{i} \hbar \theta^{i j} .
$$

Thus the scaling limit of the open string sigma-model is formally analogous to projection to the lowest Landau level for charged particles in strong uniform magnetic fields.

The more precise dynamical mechanism behind this heuristic argument can be infered from studying the two-point disk correlators deformed by the non-zero two-form $B_{i j}$, which plays the role of a magnetic flux on the worldvolume and can be turned on by a left-right asymmetric rotation of the D-brane via T-duality. The $B$-field allows one to distinguish the insertions of string fields $x^{i}(t)$ and $x^{j}\left(t^{\prime}\right)$ on the boundary of the disk in the correlation function, which depends only on the ordering of the two boundary insertion points [102]:

$$
\left\langle x^{i}(t) x^{j}\left(t^{\prime}\right)\right\rangle=-\ell_{s}^{2} G^{i j} \log \left(t-t^{\prime}\right)^{2}+\frac{\mathrm{i} \hbar}{2} \theta^{i j} \operatorname{sgn}\left(t-t^{\prime}\right),
$$

where we used the open-closed string relations

$$
\frac{1}{g+2 \pi \ell_{s}^{2} B}=\frac{1}{G}+\frac{\theta}{2 \pi \ell_{s}^{2}},
$$

with $G$ the open string metric and the bivector $\theta$ is the source of noncommutativity since it is not symmetric under interchange of $x^{i}$ with $x^{j}$. The transformation (1.9) is familiar from the Büscher rules for T-duality, with the precise connection suggested by the worldsheet approach of [55], and it explicitly determines the open string variables $(G, \theta)$ in terms of the closed string variables $(g, B)$ by

$$
G=g-\left(2 \pi \ell_{s}^{2}\right)^{2} B g^{-1} B \quad \text { and } \quad \theta=-\left(2 \pi \ell_{s}^{2}\right)^{2} G^{-1} B g^{-1} .
$$

Note that $G=g$ and $\theta=0$ exactly when $B=0$. Using this correlation function, the operator product expansion of open string tachyon vertex operators on the boundary of the disk is computed to be

$$
\mathrm{e}^{\mathrm{i} k \cdot x(t)} \cdot \mathrm{e}^{\mathrm{i} q \cdot x\left(t^{\prime}\right)}=\left(t-t^{\prime}\right)^{2 \ell_{s}^{2} k_{i} G^{i j} q_{j}} \mathrm{e}^{-\frac{\mathrm{i} \hbar}{2} k_{i} \theta^{i j} q_{j}} \mathrm{e}^{\mathrm{i}(k+q) \cdot x\left(t^{\prime}\right)}+\cdots
$$

for $t>t^{\prime}$. The second factor in (1.11) does not depend on the worldsheet coordinates and is purely a target space effect, and in the low-energy limit $\ell_{s} \rightarrow 0$ whereby $\theta=B^{-1}$, this phase factor is encoded in scattering amplitudes by the star product of fields $f, g$ given by

$$
(f \star g)(x)=\int \mathrm{d} k \int \mathrm{d} q \tilde{f}(k) \tilde{g}(q) \mathrm{e}^{-\frac{\mathrm{i} \hbar}{2} k_{i} \theta^{i j} q_{j}} \mathrm{e}^{\mathrm{i}(k+q) \cdot x}
$$

in Fourier space, which is equivalent to the formal expansion in terms of a bidifferential operator:

$$
f \star g=\cdot \exp \left(\frac{\mathrm{i} \hbar}{2} \theta^{i j} \partial_{i} \otimes \partial_{j}\right)(f \otimes g) .
$$

This is simply the Moyal-Weyl star product which is a noncommutative deformation of the pointwise product $f \cdot g$ of functions on spacetime. Its characteristic features are that it quantizes the commutator $\left[x^{i}, x^{j}\right]=\mathrm{i} \hbar \theta^{i j}$ (by defining $[f, g]_{\star}:=f \star g-g \star f$ and setting $f=x^{i}, g=x^{j}$ ), and it 
is associative: $f \star(g \star h)=(f \star g) \star h$. Since $f \star g$ differs from $f \cdot g$ by a total derivative, the star product deformation is consistent with the conformal $S L(2, \mathbb{R})$ symmetry of the worldsheet theory which leaves the cyclic ordering of boundary vertex operator insertions invariant, in the sense that the star product is 2-cyclic:

$$
\int \mathrm{d} x f \star g=\int \mathrm{d} x g \star f=\int \mathrm{d} x f \cdot g .
$$

Moreover, consistency with associativity of the operator product expansion in conformal field theory only requires crossing symmetry of the worldsheet correlation functions, which leads to the weaker 3-cyclic condition:

$$
\int \mathrm{d} x f \star(g \star h)=\int \mathrm{d} x(f \star g) \star h .
$$

It is in this way that one arrives at a noncommutative gauge theory for the massless bosonic open string modes $A_{i}$ and $\phi^{a}$; see e.g. [54, 110] for reviews and further details.

Now let us try to understand how an analogous scenario could be realised in a connection between noncommutative geometry and closed strings. Closed strings see geometry in a different way than open strings do, which from a target space perspective is due to T-duality. From the worldsheet perspective, the relevant tree-level amplitude involves correlation functions on the sphere $S^{2}$, but the situation must be different and one has to pass to higher correlators, as first pointed out by [28], since now the ordering on $S^{2}$ is ambiguous because two points can be interchanged by an $\operatorname{SL}(2, \mathbb{R})$ transformation. However, the insertion of three string fields on the sphere depends only on the relative orientation of the three points, i.e. whether the insertion of a third point lies on the same or opposite hemisphere as the other two points. A trivector flux $\theta^{i j k}$ can be used to distinguish configurations, and in analogy with the Moyal-Weyl star product (1.13) it deforms the algebra of functions with the "triproduct"

$$
f_{1} \triangle f_{2} \triangle f_{3}=\cdot \exp \left(\frac{\mathrm{i} \hbar}{6} \theta^{i j k} \partial_{i} \otimes \partial_{j} \otimes \partial_{k}\right)\left(f_{1} \otimes f_{2} \otimes f_{3}\right),
$$

which leads to a nonassociative tribracket defined by

$$
\left[f_{1}, f_{2}, f_{3}\right]_{\triangle}:=\sum_{\tau \in S_{3}} \operatorname{sgn}(\tau) f_{\tau(1)} \Delta f_{\tau(2)} \Delta f_{\tau(3)} .
$$

This quantizes the basic coordinate brackets

$$
\left[x^{i}, x^{j}, x^{k}\right]_{\triangle}=\mathrm{i} \hbar \theta^{i j k} .
$$

The purpose of these lectures is to discuss, and answer as far as possible, the following imminent questions at this stage:

(Q1) What is the trivector $\theta^{i j k}$ ?

We will see that this trivector is a 'locally non-geometric flux', called the $R$-flux. To properly discuss this, we shall have to review some ingredients of non-geometric flux compactifications, generalized geometry, and double field theory, some aspects of which are discussed in the lectures by C. Hull at this School, and which we undertake in Section 3. 
(Q2) What is the origin of the triproduct $\triangle$ ?

We will see that the nonassociativity encoded in off-shell closed string amplitudes is probed by suitable redefinitions of the coordinate fields $x^{i}$ in linear flux backgrounds. We shall find in Sections 4 and 5 that the triproduct is not the fundamental algebraic entity, but arises as the result of a non-vanishing Jacobiator for a nonassociative star product on the closed string phase space.

(Q3) Is there a nonassociative version of the closed string effective action?

Recall that the closed string effective action for the massless bosonic modes $g_{i j}, B_{i j}$ and $\Phi$ is given by

$$
S_{\text {grav }}=\frac{1}{16 \pi G} \int_{M}\left(* \operatorname{Ric}-\frac{1}{12} \mathrm{e}^{-\Phi / 3} H \wedge * H-\frac{1}{6} \mathrm{~d} \Phi \wedge * \mathrm{~d} \Phi\right) .
$$

Conformal invariance of the worldsheet theory at one-loop requires vanishing beta-functions, which are equivalent to the target space equations of motion resulting from (1.19). We shall discuss some aspects of this far-reaching future goal in Section 6, but will not provide a complete and decisive answer to the problem of the relevance of a nonassociative theory of gravity in closed string theory, which is currently a topic of ongoing investigation.

\section{A first glimpse at nonassociative geometry: Magnetic monopoles}

As we saw in the case of open strings, a simple yet instructive quantum mechanical analogue for the appearance of noncommutative geometry is provided by the motion of electric charges in background magnetic fields. A straightforward but far-reaching extension of this model likewise provides an instructive physical scenario in which to understand the appearence and implications of nonassociative geometry in the closed string sector. We shall see later on that this model has a precise analogue for closed strings propagating in locally non-geometric flux backgrounds, and it enables us to introduce some of the geometric ideas that will be used throughout this paper. The treatment of the quantum mechanical system of this section is originally due to [76, 77].

Consider the motion of a charged particle on $\mathbb{R}^{3}$ in a fixed magnetic field $\vec{B}$, possibly with sources. The kinematical momentum of the particle is $\vec{p}=m \dot{\vec{x}}$, which is the physical gaugeinvariant quantity and is not to be confused with the (gauge-variant) canonical momentum. The Hamiltonian is taken to be the kinetic energy

$$
H=\frac{\vec{p}^{2}}{2 m} .
$$

In the quantum theory, the Lorentz-Heisenberg equations of motion

$$
\dot{\vec{p}}=\frac{\mathrm{i}}{\hbar}[H, \vec{p}]=\frac{e}{2 m}(\vec{p} \times \vec{B}-\vec{B} \times \vec{p}) \quad \text { and } \quad \dot{\vec{x}}=\frac{\mathrm{i}}{\hbar}[H, \vec{x}]=\frac{\vec{p}}{m}
$$

require the deformed canonical commutation relations of a noncommutative momentum space:

$$
\left[x^{i}, x^{j}\right]=0, \quad\left[x^{i}, p_{j}\right]=\mathrm{i} \hbar \delta^{i}{ }_{j}, \quad\left[p_{i}, p_{j}\right]=\mathrm{i} \hbar e F_{i j}(\vec{x}) \quad \text { with } \quad F_{i j}=\varepsilon_{i j k} B^{k} .
$$


This formulation depends only on the magnetic field $\vec{B}$, and in particular it allows for cases in which $\nabla \cdot \vec{B} \neq 0$.

Let us understand the geometric structure underlying these commutation relations. Writing phase space coordinates collectively as $x^{I}=\left(x^{i}, p_{i}\right)$, we can express the relations in the form

$$
\left[x^{I}, x^{J}\right]=\mathrm{i} \hbar \Theta^{I J} \quad \text { with } \quad\left(\Theta^{I J}\right)=\left(\begin{array}{cc}
0 & 1_{3} \\
-1_{3} e F(\vec{x})
\end{array}\right) .
$$

The phase space bivector $\Theta=\frac{1}{2} \Theta^{I J} \partial_{I} \wedge \partial_{J}$ is not a Poisson bivector in general. The failure of the Jacobi identity for the quasi-Poisson brackets defined by $\Theta$ is controlled by the Schouten bracket

$$
[\Theta, \Theta]_{\mathrm{S}}^{I J K}=\Theta^{[\underline{I L}} \partial_{L} \Theta^{\underline{J K}]},
$$

which is the natural extension of the usual Lie bracket of vector fields to multivector fields; here only underlined indices are antisymmetrized. Then $[\Theta, \Theta]_{\mathrm{S}}=0$ if and only if the Jacobiator

$$
\left[x^{I}, x^{J}, x^{K}\right]:=\left[x^{I},\left[x^{J}, x^{K}\right]\right]+\left[x^{J},\left[x^{K}, x^{I}\right]\right]+\left[x^{K},\left[x^{I}, x^{J}\right]\right]
$$

vanishes. An easy calculation shows

$$
\left[p_{1}, p_{2}, p_{3}\right]=3 \hbar^{2} e \nabla \cdot \vec{B}=: 3 \hbar^{2} e \mu_{0} \rho_{m} .
$$

Therefore the phase space algebra of the charged particle is nonassociative in the presence of magnetic sources $\rho_{m} \neq 0$.

For source-free magnetic fields $\vec{B}$, one has $\rho_{m}=0$ and $\nabla \cdot \vec{B}=0$, so that there exists a globally defined magnetic vector potential $\vec{A}$ on $\mathbb{R}^{3}$ such that $\vec{B}=\nabla \times \vec{A}$. Then the commutation relations can be transformed to canonical form with the canonical momentum $\vec{\pi}=\vec{p}+e \vec{A}$. However, since $\vec{B}=$ $\nabla \times \vec{A}$ if and only if $\nabla \cdot \vec{B}=0$, we cannot work with canonical momenta and covariant derivatives in the presence of magnetic sources, i.e. for $\rho_{m} \neq 0$ we encounter nonassociativity and there is no linear operator $\vec{p}=\mathrm{i} \hbar \nabla-e \vec{A}$. Let us now explore how to understand magnetic sources and the ensuing violation of the Jacobi identity.

Since $\left[x^{i}, p_{j}\right]=\mathrm{i} \hbar \delta_{j}^{i}$, translations in the quantum theory are generated by the magnetic translation operators

$$
T(\vec{a})=\mathrm{e}^{\frac{\mathrm{i}}{\hbar} \vec{a} \cdot \vec{p}}
$$

with $T^{-1}(\vec{a}) \vec{x} T(\vec{a})=\vec{x}+\vec{a}$. These operators do not form a representation of the translation group on $\mathbb{R}^{3}$, as a simple calculation shows

$$
T\left(\vec{a}_{1}\right) T\left(\vec{a}_{2}\right)=\mathrm{e}^{\frac{\mathrm{i} e}{\hbar} \Phi_{2}\left(\vec{x}, \vec{a}_{1}, \vec{a}_{2}\right)} T\left(\vec{a}_{1}+\vec{a}_{2}\right),
$$

where

$$
\Phi_{2}\left(\vec{x} ; \vec{a}_{1}, \vec{a}_{2}\right)=\int_{\left\langle\vec{a}_{1}, \vec{a}_{2}\right\rangle_{\vec{x}}} \vec{B} \cdot \mathrm{d} \vec{S}
$$

is the magnetic flux through the oriented triangle $\left\langle\vec{a}_{1}, \vec{a}_{2}\right\rangle_{\vec{x}}$ based at $\vec{x} \in \mathbb{R}^{3}$ with sides $\vec{a}_{1}, \vec{a}_{2}$ and $\vec{a}_{1}+\vec{a}_{2}$. The Jacobi identity is the infinitesimal statement of associativity, and its failure results in the relations

$$
\left(T\left(\vec{a}_{1}\right) T\left(\vec{a}_{2}\right)\right) T\left(\vec{a}_{3}\right)=\mathrm{e}^{\frac{i e}{\hbar} \Phi_{3}\left(\vec{x} ; \vec{a}_{1}, \vec{a}_{2}, \vec{a}_{3}\right)} T\left(\vec{a}_{1}\right)\left(T\left(\vec{a}_{2}\right) T\left(\vec{a}_{3}\right)\right),
$$


where

$$
\Phi_{3}\left(\vec{x} ; \vec{a}_{1}, \vec{a}_{2}, \vec{a}_{3}\right)=\int_{\partial\left\langle\vec{a}_{1}, \vec{a}_{2}, \vec{a}_{3}\right\rangle_{\vec{x}}} \vec{B} \cdot \mathrm{d} \vec{S}=\int_{\left\langle\vec{a}_{1}, \vec{a}_{2}, \vec{a}_{3}\right\rangle_{\vec{x}}} \nabla \cdot \vec{B} \mathrm{~d} V
$$

is the magnetic charge $q_{m}$ enclosed by the oriented tetrahedron $\left\langle\vec{a}_{1}, \vec{a}_{2}, \vec{a}_{3}\right\rangle_{\vec{x}}$ based at $\vec{x} \in \mathbb{R}^{3}$ with sides $\vec{a}_{1}, \vec{a}_{2}, \vec{a}_{3}, \vec{a}_{1}+\vec{a}_{2}, \vec{a}_{2}+\vec{a}_{3}$ and $\vec{a}_{1}+\vec{a}_{2}+\vec{a}_{3}$, and we have used the divergence theorem.

It follows that associativity of translations is ensured when [76]

$$
\frac{\mu_{0} e q_{m}}{\hbar} \in 2 \pi \mathbb{Z}
$$

which is the celebrated Dirac charge quantization condition. Then the usual quantum mechanical formalism can be applied with linear operators on a separable Hilbert space. But this restricts the form of the magnetic field $\vec{B}$, which must be sourced by a point-like magnetic monopole (or a collection thereof), so that the phase $\Phi_{3}$ does not lose its integrality when the translation vectors $\vec{a}_{i}$ are continuously varied. In this case the magnetic source must lie either inside or outside the tetrahedron $\left\langle\vec{a}_{1}, \vec{a}_{2}, \vec{a}_{3}\right\rangle_{\vec{x}}$, and is given by the Dirac monopole field

$$
\vec{B}=\mu_{0} q_{m} \frac{\vec{x}}{|\vec{x}|^{3}} \quad \text { with } \quad \nabla \cdot \vec{B}=4 \pi \mu_{0} q_{m} \delta(\vec{x}) .
$$

What becomes of the Jacobi identity in this case? We note that it is violated precisely at the loci of the magnetic charges, which for Dirac monopoles occur at isolated points and so can be excised from $\mathbb{R}^{3}$, where the magnetic field $\vec{B}$ is singular. Such an excision is also natural from the point of view of angular momentum conservation, which imples that the electric charges never reach the monopoles and their wavefunction vanishes at the monopole locations [16]. This leads to a geometric description of Dirac monopoles in terms of connections on a non-trivial $U(1)$ bundle $P \rightarrow \mathbb{R}^{3} \backslash\{\overrightarrow{0}\} \simeq S^{2}$ of first Chern class $c_{1}(P)=\mu_{0} e q_{m} / 2 \pi \hbar$, and the wavefunctions of the particle live in the Hilbert space of square-integrable sections of $P$. In this case the map $\vec{a} \mapsto T(\vec{a})$ defines a projective representation of the translation group of $\mathbb{R}^{3} \backslash\{\overrightarrow{0}\}$ on this Hilbert space, and the projective phase $\mathrm{e}^{\frac{i e}{\hbar} \Phi_{2}}$ is the group two-cocycle of the representation.

For our later considerations we are interested in situations corresponding to a constant homogeneous magnetic charge density background $\rho_{m}$, whose algebraic structure was first studied in [62]. The analogue of the rotationally symmetric field (2.14) in this case is given by

$$
\vec{B}=\frac{\mu_{0} \rho_{m}}{3} \vec{x}
$$

The magnetic charge is now uniformly distributed over all space, so that the phase space coordinate algebra becomes everywhere nonassociative. In this case removing the magnetic sources from $\mathbb{R}^{3}$ would leave an empty space. In this sense the momentum space of an electric charge in a uniform magnetic charge distribution is 'locally non-geometric'. The constant magnetic charge density is not described by a connection on a $U(1)$-bundle over $\mathbb{R}^{3} \backslash\{\overrightarrow{0}\}$, but rather in terms of a connection on a (trivial) $U(1)$-gerbe on $\mathbb{R}^{3}$, i.e. by a " $B$-field"

$$
F_{i j}=\varepsilon_{i j k} B^{k}=\frac{\mu_{0} \rho_{m}}{3} \varepsilon_{i j k} x^{k}
$$


with curvature $H=\mathrm{d} F=\mu_{0} \rho_{m} \mathrm{~d} x^{1} \wedge \mathrm{d} x^{2} \wedge \mathrm{d} x^{3}$; since $H \neq 0$ everywhere there is not even a local magnetic vector potential $\vec{A}$ in this instance. Moreover, now the phase factors $\Phi_{2}$ yield twocochains, rather than two-cocycles, whose coboundary is the phase $\mathrm{e}^{\frac{\mathrm{i} e}{\hbar} \Phi_{3}}$ which hence defines a three-cocycle of the translation group of $\mathbb{R}^{3}$. Thus the quantum theory with this three-cocycle nessecitates nonassociative quantum mechanics. Geometrically, the bivector $\Theta$ in this case defines a 'twisted Poisson structure' on phase space. As we will see later on, such a quantum system makes perfect physical sense and possesses fascinating properties.

This simple quantum mechanical example illustrates the string theoretical considerations which will follow, in the topic of non-geometric flux compactifications that we turn to next. Our string theory considerations will lead naturally to an approach to nonassociative geometry via deformation quantization, analogously to the open string case, which will be the main tool of this paper. However, as phase space quantum mechanics comes with its own issues, as we discuss later, let us point out for completeness some alternative approaches to the nonassociative quantum mechanics alluded to above, of which there are currently three that each have their own deficiencies as well.

Firstly, and most straightforwardly, one may generalize the technique of symplectic realization from Poisson geometry to the twisted Poisson structure $\Theta$ and embed the nonassociative phase space into a symplectic manifold of twice the original dimension [82]. In this associative framework standard techniques of geometric or canonical quantization are available, and in particular a global magnetic vector potential exists on the doubled configuration space; its drawback is that, while the doubling is tantalizing reminescent of the framework of double field theory discussed below, it is not possible to eliminate the spurious auxiliary degrees of freedom that enable the reformulation in terms of associative geometry. Secondly, one can exploit the geometric structure of the gerbe associated to the distribution of magnetic charge to face the nonassociativity head on and define quantum states that live in the 2-Hilbert space of sections of this gerbe, in analogy to the ordinary Hilbert space of sections of a line bundle in the source-free case [114, 39]. This gives a geometric description of the magnetic translation operators (2.8) acting on this 2-Hilbert space with the three-cocycle above interpreted as a higher projective phase of a 2-representation; the drawback of this approach is that it is rather technically complicated and it is difficult to represent observables such as the Hamiltonian operator (2.1) on the 2-Hilbert space, while conceptually it is not clear what is the meaning of such higher quantum states. Thirdly, one can apply transgression techniques to map the gerbe to a line bundle over the loop space of the configuration manifold [98, 99, 40]. This approach is naturally suggested by the closed string origin of nonassociativity, and with it one can apply standard techniques of geometric quantization on loop space which successfully captures some predictions of string theory; the drawback of this approach is that it requires difficult infinitedimensional analysis which makes computations of physical quantities, such as expectation values, seemingly intractable. Hence in all approaches one trades nonassociativity for some other sort of technical or conceptual complication.

\section{Non-geometric fluxes and nonassociative geometry}

The purpose of this section is to explain what the notion of "non-geometry" means in string theory, and in particular to answer Question (Q1) from Section 1. Recall that in closed string theory, in addition to the spacetime metric $g_{i j}$, there is a massless Kalb-Ramond two-form $B$ in the 
NS-NS sector with curvature three-form $H=\mathrm{d} B$. The motion of the strings is described by the two-dimensional non-linear sigma-model action (1.4). The classical vacua are described by twodimensional conformal field theories, and in this setting the target space geometry is emergent. On the other hand, there exist conformal field theories which cannot be identified with such simple large radius geometries, such as left-right asymmetric orbifolds, and in such instances we will advocate the point of view that the target space interpretation is related to noncommutative and nonassociative geometry. This is the case when a gauge flux is turned on in the worldvolume field theory of a D-brane, which corresponds to an asymmetric rotation in the boundary conformal field theory.

Another left-right asymmetric worldsheet operation is that of T-duality, which reflects rightmoving strings while leaving the left-moving sector unchanged. From the perspective of a $d$ dimensional target space, a T-duality $\mathscr{T}_{i}$ along the $i$-th direction exchanges momentum modes $p_{i}$ with winding modes $w^{i}$, and correspondingly for the canonically conjugate variables: position coordinates $x^{i}$ are exchanged with their dual "winding coordinates" $\tilde{x}_{i}$. The collection of symmetry transformations form the split-signature orthogonal group $O(d, d)$ which is the continuous extension of the physical T-duality group $O(d, d ; \mathbb{Z})$ of toroidally compactified closed string theory.

String theory with fluxes is of interest both for its geometric allure and because of its relevance to observable phenomenology and cosmology (see e.g. [60, 53, 32] for reviews): Flux compactifications can lead to generalized geometric structures, obtained for example by patching together with string symmetries, while at the same time they stabilize moduli on the string landscape. They are also of importance in the AdS/CFT correspondence. Starting from flat space with non-vanishing NS-NS $H$-flux, T-duality gives rise to a chain of geometric and non-geometric fluxes [71, 107]

$$
H_{i j k} \stackrel{\mathscr{T}_{i}}{\longrightarrow} f_{j k}^{i} \stackrel{\mathscr{T}_{j}}{\longrightarrow} Q^{i j}{ }_{k} \stackrel{\mathscr{T}_{k}}{\longrightarrow} R^{i j k}
$$

Let us briefly describe the geometrical meaning of each member of this T-duality chain. The first term is of course the geometric NS-NS flux $H=\mathrm{d} B$, which represents the characteristic class of a $U(1)$-gerbe by generalized Dirac quantization of fluxes in string theory. The second term is a metric flux, which appears as torsion in the geometry: In a suitable basis of vielbeins $e^{i}$, with inverses $e_{i}$, it appears in the Maurer-Cartan equations $\mathrm{d} e^{i}=-\frac{1}{2} f^{i}{ }_{j k} e^{j} \wedge e^{k}$, or equivalently as the structure constants of the non-trivial Lie bracket of vector fields $\left[e_{i}, e_{j}\right]=f^{k}{ }_{i j} e_{k}$. The third member is the first example of a non-geometric frame, and $Q^{i j}{ }_{k}$ is called a 'globally non-geometric' $Q$-flux. These are also called T-folds, which have a local description in terms of conventional Riemannian geometry, but globally the transition functions between local charts also involve T-duality transformations. The final member of the chain is the most mysterious frame and the one which shall occupy most of our attention: Here $R^{i j k}$ is called a 'locally non-geometric' $R$-flux. This background cannot even be described locally by conventional geometry and has no clear target space interpretation. As we discuss below it is this frame that this gives rise to a nonassociative geometry.

Describing these fluxes and understanding this non-geometric regime of string theory requires generalized geometry and its extension to double field theory, which is an $O(d, d)$-symmetric theory treating the metric and $B$-field on equal footing, and which is covered in the lectures by C. Hull at this School. 


\subsection{Generalized geometry}

The geometric and non-geometric fluxes appearing in the T-duality chain (3.1) can be regarded as structure constants of a generalized bracket

$$
\begin{aligned}
& {\left[e_{i}, e_{j}\right]_{\mathrm{R}}=f^{k}{ }_{i j} e_{k}+H_{i j k} e^{k},} \\
& {\left[e^{i}, e_{j}\right]_{\mathrm{R}}=f^{i}{ }_{j k} e^{k}-Q^{i k}{ }_{j} e_{k},} \\
& {\left[e^{i}, e^{j}\right]_{\mathrm{R}}=Q^{i j}{ }_{k} e^{k}+R^{i j k} e_{k},}
\end{aligned}
$$

for a local vielbein basis of sections $\left(e_{i}, e^{i}\right)$ of the vector bundle $E=T M \oplus T^{*} M$, which in generalized geometry is called the generalized tangent bundle [66,61]. In this form the bracket is usually called the Roytenberg bracket $[95,65,31]$, and its reductions for various choices of vanishing fluxes gives the usual Courant and Dorfman brackets of generalized geometry. It governs the worldsheet current algebras in flux compactifications of string theory, see e.g. [2, 64, 38].

The sections of the generalized tangent bundle $E=T M \oplus T^{*} M$ are denoted as $X+\xi$ with $X=X^{i} e_{i}$ a vector field and $\xi=\xi_{i} e^{i}$ a one-form on $M$. The bundle $E$ carries a canonical $O(d, d)$ structure through the natural pairing $\left\langle e_{i}, e^{j}\right\rangle=\delta_{i}{ }^{j}$ of the tangent bundle $T M$ with the cotangent bundle $T^{*} M$ of the target space $M$. The structure group $O(d, d)$ has two natural abelian subgroups acting on sections of the generalized tangent bundle in the following way:

- $\underline{B \text {-transforms: }}\left(\begin{array}{cc}1_{d} & 0 \\ B & 1_{d}\end{array}\right): X+\xi \longmapsto X+\left(\xi+l_{X} B\right)$

- $\underline{\theta \text {-transforms: }}\left(\begin{array}{cc}1_{d} & \theta \\ 0 & 1_{d}\end{array}\right): X+\xi \longmapsto\left(X+\theta^{\sharp} \xi\right)+\xi$,

where $B$ is a two-form and $\theta$ is a bivector which induce the natural contraction maps $\imath: T M \rightarrow T^{*} M$, $\left(l_{X} B\right)_{i}=B_{i j} X^{j}$ and $\theta^{\sharp}: T^{*} M \rightarrow T M,\left(\theta^{\sharp} \xi\right)^{i}=\theta^{i j} \xi_{j}$. Any $O(d, d)$-transformation $\mathscr{O} \in O(d, d)$ can be written in the form

$$
\mathscr{O}=\left(\begin{array}{cc}
1_{d} & 0 \\
B & 1_{d}
\end{array}\right)\left(\begin{array}{cc}
N & 0 \\
0 & N^{-\top}
\end{array}\right)\left(\begin{array}{cc}
1_{d} & \theta \\
0 & 1_{d}
\end{array}\right) \text { with }\left(\begin{array}{cc}
N & 0 \\
0 & N^{-\top}
\end{array}\right): X+\xi \longmapsto t_{X} N+\left(N^{-\top}\right)^{\sharp} \xi
$$

where $N \in G L(d)$ determines a general linear transformation of sections.

The generalization of the Lie bracket of vector fields $X, Y$ on $T M$ to sections $X+\xi, Y+\eta$ of $T M \oplus T^{*} M$ is provided by the Dorfman bracket

$$
[X+\xi, Y+\eta]_{\mathrm{D}}=[X, Y]+£_{X} \eta-\imath_{Y} \mathrm{~d} \xi
$$

where $£_{X}$ denotes the Lie derivative along $X$. Fluxes can be incorporated into this bracket structure by adding appropriate twisting terms, e.g. $l_{X} l_{Y} H$. Alternatively, they may be added by applying suitable $O(d, d)$-transformations of sections of the generalized tangent bundle, starting from the standard geometric frame with metric flux, basis $\left(e_{i}, e^{i}\right)$ and the Dorfman brackets

$$
\left[e_{i}, e_{j}\right]_{\mathrm{D}}=f^{k}{ }_{i j} e_{k}, \quad\left[e^{i}, e_{j}\right]_{\mathrm{D}}=f^{i}{ }_{j k} e^{k} \quad \text { and } \quad\left[e^{i}, e^{j}\right]_{\mathrm{D}}=0 .
$$

Then under a $B$-transform of the basis $\left(e_{i}, e^{i}\right)$ these brackets map into

$$
\left[e_{i}, e_{j}\right]_{\mathrm{R}}=f^{k}{ }_{i j} e_{k}+H_{i j k} e^{k}, \quad\left[e^{i}, e_{j}\right]_{\mathrm{R}}=f_{j k}^{i} e^{k} \quad \text { and } \quad\left[e^{i}, e^{j}\right]_{\mathrm{R}}=0,
$$


with the geometric NS-NS flux $H=\mathrm{d} B$, while under a $\theta$-transform they map to

$$
\left[e_{i}, e_{j}\right]_{\mathrm{R}}=f^{k}{ }_{i j} e_{k}, \quad\left[e^{i}, e_{j}\right]_{\mathrm{R}}=f^{i}{ }_{j k} e^{k}-Q^{i k}{ }_{j} e_{k} \quad \text { and } \quad\left[e^{i}, e^{j}\right]_{\mathrm{R}}=Q^{i j}{ }_{k} e^{k}+R^{i j k} e_{k},
$$

with the globally and locally non-geometric fluxes

$$
\left.Q_{k}^{i j}=\partial_{k} \theta^{i j}+2 f^{[i}{ }_{k l} \theta^{l j} \underline{\underline{j}}\right] \quad \text { and } \quad R^{i j k}=3\left([\theta, \theta]_{\mathrm{S}}^{i j k}+f^{\left[\underline{i}_{l m}\right.} \theta^{\underline{j} m} \theta^{\underline{k} m]}\right) .
$$

In this way the local $O(d, d)$-transformations of the Dorfman bracket (3.5) reproduce the Roytenberg bracket (3.2). What is particularly noteworthy and relevant for us here is that the nongeometric fluxes are determined by a bivector $\theta$.

\subsection{Double field theory}

Let us now briefly explain how double field theory [108, 109, 73] provides a microscopic description of $Q$-flux and $R$-flux, through the chain (3.1) of T-duality transformations, by a formal definition and unified description of non-geometric fluxes; see e.g. [1, 22, 69] for reviews. The idea of double field theory is to double the target space coordinates $x^{i}$ to $x^{I}=\left(x^{i}, \tilde{x}_{i}\right)$, where $\tilde{x}_{i}$ are the T-dual "winding coordinates". This gives a formalism with manifest $O(d, d)$-symmetry that allows one to perform such T-dualities to non-geometric frames.

Double field theory is a field theory for the massless modes of closed bosonic string theory that treats diffeomorphism symmetry and $B$-field gauge transformations on equal footing by assembling them into the generalized metric

$$
\mathscr{H}=\left(\begin{array}{cc}
g^{-1} & -2 \pi \ell_{s}^{2} g^{-1} B \\
2 \pi \ell_{s}^{2} B g^{-1} & g-\left(2 \pi \ell_{s}^{2}\right)^{2} B g^{-1} B
\end{array}\right) .
$$

This metric can be written in terms of a Schur decomposition

$$
\mathscr{H}=\left(\begin{array}{cc}
1_{d} & 0 \\
2 \pi \ell_{s}^{2} B & 1_{d}
\end{array}\right)\left(\begin{array}{cc}
g^{-1} & 0 \\
0 & g
\end{array}\right)\left(\begin{array}{cc}
1_{d} & -2 \pi \ell_{s}^{2} B \\
0 & 1_{d}
\end{array}\right),
$$

which identifies it as a $B$-transform of the doubled space metric when $B=0$. There is a global $O(d, d)$-symmetry that includes T-duality and acts as

$$
x^{I} \longmapsto \mathscr{O}_{J}^{I} x^{J}, \quad \mathscr{H} \longmapsto \mathscr{O}^{\top} \mathscr{H} \mathscr{O} \quad \text { with } \quad \mathscr{O}=\left(\mathscr{O}_{J}^{I}\right) \in O(d, d)
$$

One then constructs an $O(d, d)$-invariant action for $\mathscr{H}$ and halves the degrees of freedom by imposing the strong constraint $\partial_{i} \otimes \tilde{\partial}^{i}+\tilde{\partial}^{i} \otimes \partial_{i}=0$ on all products of fields of double field theory, which is a strong version of the level-matching condition $L_{0}-\bar{L}_{0}=0$ in the worldsheet conformal field theory, i.e. $p_{i} w^{i}=0$. Solving the strong constraint amounts to choosing a polarization on the doubled space [72]; for example, in the geometric supergravity frame one takes $\tilde{\partial}^{i}:=\frac{\partial}{\partial \tilde{x}_{i}}=0$, which reduces the fields of double field theory to fields of generalized geometry. Different polarisations define different T-duality frames, and any two frames are related by an $O(d, d)$-transformation.

Let us consider the example of flat space, $g^{\circ}=1_{d}$, with constant $H$-flux, and choose the KalbRamond field in the symmetric gauge $B_{i j}^{\circ}=\frac{1}{3} H_{i j k} x^{k}$; this is locally defined (for $x \in \mathbb{R}^{d}$ ), but not 
globally if the spacetime is e.g. a torus, where it is only defined up to large gauge transformations. A T-duality in the $i$-th direction interchanges $x^{i}$ with $\tilde{x}_{i}$ and is implemented by the factorized $O(d, d)$-transformation matrix

$$
\mathscr{T}_{i}=\left(\begin{array}{cc}
1_{d}-E_{i} & E_{i} \\
E_{i} & 1_{d}-E_{i}
\end{array}\right),
$$

where $E_{i}$ are the $d \times d$ matrix units $\left(E_{i}\right)_{k l}=\delta_{k i} \delta_{l i}$. Consider now the effect of applying two successive T-duality transformations $\mathscr{T}_{(i j)}:=\mathscr{T}_{i} \mathscr{T}_{j}$ to the corresponding generalized metric:

$$
\begin{aligned}
\mathscr{H}_{(i j)} & =\mathscr{T}_{(i j)}^{\top}\left(\begin{array}{cc}
1_{d} & -2 \pi \ell_{s}^{2} B^{\circ} \\
2 \pi \ell_{s}^{2} B^{\circ} & 1_{d}-\left(2 \pi \ell_{s}^{2}\right)^{2}\left(B^{\circ}\right)^{2}
\end{array}\right) \mathscr{T}_{(i j)} \\
& =:\left(\begin{array}{cc}
g^{-1} & -2 \pi \ell_{s}^{2} g^{-1} B \\
2 \pi \ell_{s}^{2} B g^{-1} & g-\left(2 \pi \ell_{s}^{2}\right)^{2} B g^{-1} B
\end{array}\right) .
\end{aligned}
$$

One easily computes that the new metric and Kalb-Ramond field $(g, B)$ are not globally defined in the directions orthogonal to the $\left(x^{i}, x^{j}\right)$-plane, which is the earmark of the 'global non-geometry' of the T-fold in the geometric parameterization. However, a suitable field redefinition appropriate to the transformation from a geometric frame to a non-geometric frame yields a new parameterization of the generalized metric in double field theory as [6]

$$
\mathscr{H}_{(i j)}=\left(\begin{array}{cc}
G^{-1}-\frac{1}{\left(2 \pi \ell_{s}^{2}\right)^{2}} \theta G \theta & \frac{1}{2 \pi \ell_{s}^{2}} \theta G \\
-\frac{1}{2 \pi \ell_{s}^{2}} G \theta & G
\end{array}\right),
$$

where the new metric $G$ and bivector $\theta=\frac{1}{2} \theta^{i j} \partial_{i} \wedge \partial_{j}$ are given precisely by the open-closed string relation (1.9), as anticipated from the Büscher rules. This metric can similarly be obtained from a $\theta$-transform given by

$$
\mathscr{H}_{(i j)}=\left(\begin{array}{cc}
1_{d} & \frac{1}{2 \pi \ell_{s}^{2}} \theta \\
0 & 1_{d}
\end{array}\right)\left(\begin{array}{cc}
G^{-1} & 0 \\
0 & G
\end{array}\right)\left(\begin{array}{cc}
1_{d} & 0 \\
-\frac{1}{2 \pi \ell_{s}^{2}} \theta & 1_{d}
\end{array}\right) .
$$

In the present case one computes

$$
G=1_{d} \quad \text { and } \quad \theta^{i j}=Q^{i j}{ }_{k} x^{k},
$$

which defines flat space with constant non-geometric $Q$-flux

$$
Q^{i j}{ }_{k}=\partial_{k} \theta^{i j}
$$

Finally, let us apply a T-duality transformation $\mathscr{T}_{k} \in O(d, d)$ along a remaining non-isometric direction, which exchanges the physical coordinate $x^{k}$ with the winding coordinate $\tilde{x}_{k}$ and maps the geometric data (3.16) to

$$
\tilde{G}=1_{d} \quad \text { and } \quad \tilde{\theta}^{i j}=R^{i j k} \tilde{x}_{k} .
$$

This defines flat space with constant non-geometric $R$-flux

$$
R^{i j k}=\tilde{\partial}^{[i} \tilde{\theta}^{j k]} .
$$

As the $R$-flux explicitly involves derivatives of the winding coordinates, it cannot be described in ordinary geometry and in this sense the $R$-flux frame is 'locally non-geometric'. In this simple example, one can alternatively regard $\tilde{\theta}$ as a two-form on the dual winding space of curvature $R=\mathrm{d} \tilde{\theta}$. 


\subsection{Overview of conformal field theory results}

Let us now summarise the explicit worldsheet calculations which have suggested the appearance of noncommutative and nonassociative deformations of geometry in the non-geometric frames of closed string theory:

- The original suggestion of nonassociativity by [28] computes the cyclic equal time double commutator

$$
\left[x^{i}, x^{j}, x^{k}\right]:=\lim _{\sigma_{i} \rightarrow \sigma}\left[\left[x^{i}\left(\sigma_{1}, \tau\right), x^{j}\left(\sigma_{2}, \tau\right)\right], x^{k}\left(\sigma_{3}, \tau\right)\right]+\text { cyclic }
$$

in the $S U(2)$ Wess-Zumino-Witten model with $H$-flux, and finds a non-vanishing target space quantity.

- Explicit calculations of phase space commutators by canonical quantization of closed strings in flat space and in a linear $B$-field background were carried out in [84, 46, 5], by studying monodromy properties and the corresponding twisted closed string boundary conditions, which lead to a shifted closed string mode expansion analogous to the open string case and expansions in asymmetric orbifold string theories. These calculations reveal generally a doubled phase space nonassociative geometry in the different T-duality frames.

- Correlators of products of tachyon vertex operators in sigma-model perturbation theory about a flat geometry with small constant $H$-flux were computed in [33], and after conformal field theory T-duality shown to reproduce the triproducts discussed in Section 1.

Further evidence is provided in e.g. [42, 47, 24, 17, 93].

The resulting quantum geometry structures can be described as follows. Let us first consider the $Q$-flux frame. Naively, in analogy with the open string case, the bivector of (3.16) in the non-geometric parameterization would suggest noncommutativity, but in the closed string case one needs more: Only closed strings which wind in the $Q$-flux frame can probe a quantum deformation of the geometry, and the noncommutativity in this case is determined by a Wilson line of the $Q$-flux as [7]

$$
\left[x^{i}, x^{j}\right]=\frac{\mathrm{i} \ell_{s}^{4}}{3 \hbar} \oint \mathrm{d} \theta^{i j}=\frac{\mathrm{i} \ell_{s}^{4}}{3 \hbar} \oint Q^{i j}{ }_{k} \mathrm{~d} x^{k}=\frac{\mathrm{i} \ell_{s}^{4}}{3 \hbar} Q^{i j}{ }_{k} w^{k}
$$

where $w^{k}$ are the closed string winding numbers. Since the winding numbers are central elements in this algebra, i.e. $\left[w^{i}, w^{j}\right]=\left[x^{i}, w^{j}\right]=0$, these relations define a noncommutative but associative geometry.

To probe nonassociativity one needs to introduce local $R$-flux. The explicit computations above yield the tribracket

$$
\left[x^{i}, x^{j}, x^{k}\right]=\ell_{s}^{4} R^{i j k} .
$$

Let us quickly sketch how this bracket is derived from conformal perturbation theory, refering to the original work [33] and also to the nice review [26] for details of the calculation. Using complex coordinates on the Riemann sphere $S^{2} \simeq \mathbb{C} \cup\{\infty\}$, the worldsheet equations of motion to 
linear order in the $H$-flux for a flat target space read $\partial \bar{\partial} x^{i}=\frac{1}{2} H^{i}{ }_{j k} \partial x^{j} \bar{\partial} x^{k}$, and thus the coordinate fields have to be modified in order to be consistent with the conformal field theory description, wherein the perturbation of the free worldsheet sigma-model by the $H$-flux should yield a marginal deformation. We therefore replace the usual conserved currents by

$$
\mathscr{J}^{i}=\mathrm{i} \partial x^{i}-\frac{\mathrm{i}}{2} H^{i}{ }_{j k} \partial x^{j} x_{\mathrm{r}}^{k} \quad \text { and } \quad \overline{\mathscr{J}}^{i}=\mathrm{i} \bar{\partial} x^{i}-\frac{\mathrm{i}}{2} H_{j k}^{i} x_{1}^{j} \bar{\partial} x^{k}
$$

where $x^{i}=x_{1}^{i}+x_{\mathrm{r}}^{i}$ is the decomposition of the string fields into left and right moving modes; the dual winding fields are then given by $\tilde{x}^{i}=x_{1}^{i}-x_{\mathrm{r}}^{i}$. The correlation functions of three insertions of the currents $\mathscr{J}^{i}$ are readily computed, and after writing $\mathscr{J}^{i}=: \mathrm{i} \partial \mathscr{X}^{i}$ and performing three worldsheet integrations, one arrives at the closed string generalization of the second term in the open string propagator (1.8) for the modified string fields $\mathscr{X}^{i}$ with the sign function, that arises from combinations of the complex logarithm function, replaced by certain combinations of the complex Rogers dilogarithm function, and the bivector $\theta^{i j}$ substituted by the trivector $\theta^{i j k}=\frac{\ell_{s}^{4}}{\hbar} H^{i j k}$.

A triple T-duality transformation $\mathscr{T}_{(i j k)}:=\mathscr{T}_{i} \mathscr{T}_{j} \mathscr{T}_{k}$ is affected in the worldsheet conformal field theory as an asymmetric reflection of the right-moving string coordinates: $\mathscr{X}_{1}^{i} \mapsto \mathscr{X}_{1}^{i}, \mathscr{X}_{\mathrm{r}}^{i} \mapsto$ $-\mathscr{X}_{\mathrm{r}}^{i}$, which maps winding modes $w^{i}$ in the $H$-flux frame to momentum modes $p_{i}$ in the $R$-flux frame. The three-point correlators of the corresponding tachyon vertex operators $\mathrm{e}^{\mathrm{i} k \cdot \mathscr{X}}$ thereby produce a phase which is trivial in the $H$-flux frame, but which is non-trivial in the $R$-flux frame and encoded in scattering amplitudes

$$
(f \triangle g \triangle h)(x)=\int \mathrm{d} k \int \mathrm{d} q \int \mathrm{d} r \tilde{f}(k) \tilde{g}(q) \tilde{h}(r) \mathrm{e}^{-\frac{\mathrm{i} t_{s}^{4}}{6} R^{i j k} k_{i} q_{j} r_{k}} \mathrm{e}^{\mathrm{i}(k+q+r) \cdot x}
$$

by the triproducts (1.16) with $\theta^{i j k}=\frac{\ell_{s}^{4}}{\hbar} R^{i j k}$. These phases are consistent with the crossing symmetry of conformal field theory correlation functions, or equivalently associativity of the operator product expansion, because by momentum conservation one has $p_{i} q_{j} r_{k} \theta^{i j k}=0$ whenever $p+q+r=0$. This is equivalent to the cyclicity property of the triproduct

$$
\int \mathrm{d} x f \triangle g \triangle h=\int \mathrm{d} x f \cdot g \cdot h,
$$

which for constant $R$-flux follows from integration by parts since the two integrands differ by total derivatives. Hence the triproducts are consistent with the axioms of conformal field theory and nonassociativity is not probed by the on-shell theory. Note that it is the modified string coordinates $\mathscr{X}^{i}$ and not the original ones $x^{i}$ which probe the nonassociative geometry, a feature which is also confirmed in other worldsheet approaches [17].

Alternatively, following what we did for the $Q$-flux frame, using the bivector from (3.18) we find noncommutativity probed by closed strings which propagate in the $R$-flux frame given by

$$
\left[x^{i}, x^{j}\right]=\frac{\mathrm{i} \ell_{s}^{4}}{3 \hbar} \oint \mathrm{d} \tilde{\theta}^{i j}=\frac{\mathrm{i} \ell_{s}^{4}}{3 \hbar} \oint R^{i j k} \mathrm{~d} \tilde{x}_{k}=\frac{\mathrm{i} \ell_{s}^{4}}{3 \hbar} R^{i j k} p_{k}
$$

where $p_{k}$ are the closed string momentum modes. However, now the variables $x^{i}$ and $p_{i}$ are canonically conjugate, and so the tribracket (3.22) can be regarded as the Jacobiator of the precursor nonassociative phase space algebra

$$
\left[x^{i}, x^{j}\right]=\frac{\mathrm{i} \ell_{s}^{4}}{3 \hbar} R^{i j k} p_{k}, \quad\left[x^{i}, p_{j}\right]=\mathrm{i} \hbar \delta^{i}{ }_{j} \quad \text { and } \quad\left[p_{i}, p_{j}\right]=0 .
$$


Note that (3.27) for $d=3$ is formally the same as the magnetic monopole phase space algebra (2.3) in the case of the linear magnetic field (2.15), after applying the magnetic duality transformation of order four given by

$$
x^{i} \longmapsto-p_{i}, \quad p_{i} \longmapsto x^{i} \quad \text { and } \quad \mu_{0} \rho_{m} \hbar e \varepsilon_{i j k} \longmapsto-\frac{\ell_{s}^{4}}{\hbar} R^{i j k},
$$

which in the absence of fluxes defines a canonical transformation. In particular, the locally nongeometric flux $R$ now appears as the curvature three-form of a two-form connection on a gerbe over momentum space [89]. The quantization of the brackets (3.27) can be captured by an explicit nonassociative star product on the phase space $T^{*} M$ of the original target space $M[89,16,83]$, whose construction and physical implications will be discussed in detail later on.

\subsection{Caveats}

There are a few loopholes in the conformal field theory derivations mentioned above that should be pointed out:

1. The conformal field theory calculations are all performed in flat space with constant $H$-flux and constant dilaton. Such a background only satisfies the closed string equations of motion derived from the action (1.19) to linear order in $H$ (in the critical dimension):

$$
\begin{aligned}
& 0=\operatorname{Ric}_{i j}-\frac{1}{4} H_{i}{ }^{j k} H_{j k l}+2 \nabla_{i} \nabla_{j} \Phi+O\left(\ell_{s}^{4}\right), \\
& 0=-\frac{1}{2} \nabla_{k} H^{k}{ }_{i j}+\ell_{s}^{2} H_{i j}{ }^{k} \nabla_{k} \Phi+O\left(\ell_{s}^{4}\right), \\
& 0=\ell_{s}^{2}\left((\nabla \Phi)^{2}-\frac{1}{2} \nabla^{2} \Phi-\frac{1}{24} H^{2}\right)+O\left(\ell_{s}^{4}\right) .
\end{aligned}
$$

2. On a compact space with non-trivial topology such as the torus, the $H$-flux is quantized by virtue of generalized Dirac charge quantization in string theory. The true T-duality transformations connecting physically equivalent string theories are then valued in the discrete subgroup $O(d, d ; \mathbb{Z}) \subset O(d, d)$. In this case the notion of linear order in $H$, as well as its extrapolation to non-geometric polarizations, is meaningless as $H$ cannot be continuously varied.

3. The triproduct violates the strong constraint between the background $R^{i j k}$ and the fluctuations around it [34]. This is not a serious problem if polarization can be achieved by a weaker constraint; this issue is currently under debate as the strong constraint indeed seems too stringent for some considerations. We elaborate on this point further in Section 5.

4. Nonassociativity does not appear in the algebra of conformal currents of the worldsheet theory, but rather through non-conformal fields such as $\mathscr{X}^{i}$ above.

5. Thus far no construction is available of $H$-deformed graviton vertex operators, whose correlation functions would clarify if and how a nonassociative deformation of the gravity theory defined by (1.19) could be of relevance in closed string theory. 
Despite these caveats, the geometric structures unveiled and their novel physical implications are so rich and beautiful that work has plugged along in this direction to further explore the ramifications and relevance of nonassociative geometry in closed string theory. For example, recent discussions reveal that Yang-Baxter deformations (in general a class of non-abelian T-duality) may be viewed as the open-closed string map (1.9) at the generalized supergravity level, see [18] and references therein; in this setting the Jacobi identity yields the classical Yang-Baxter equation for the isometry group of the background. In the remainder of this contribution we shall discuss further ways of naturally understanding the origins of nonassociativity, and then proceed to unravel some of the physics of this structure.

\section{Higher geometrization of non-geometry}

In Section 3 we have discussed two geometric ways of making sense of the globally and locally non-geometric frames of flux compactifications: One through an extension of geometry into the realm of generalized geometry and double field theory, and the other through noncommutative and nonassociative deformations of the closed string phase space. To better understand the relationship between these two points of view from a dynamical perspective, we shall now show that the nongeometry of string backgrounds is geometrized through a membrane sigma-model, suggesting that the proper probes of these backgrounds should be open membranes whose boundary modes are the closed string degrees of freedom. Such an approach was first suggested by [65], and developed in detail in [89]; see [43, 23] for further developments. In Section 5 below we shall show that the quantization of this sigma-model produces the nonassociative phase space star product that we have been advertising.

To understand the idea behind this framework, let us recall how we treated the open string sigma-model (1.4). In the Seiberg-Witten scaling limit it reduces to the action (1.5), whose first order formalism describes a two-dimensional topological field theory called the 'Poisson sigmamodel'. As we will discuss in Section 5, the quantization of this sigma-model reproduces the commutators (1.7) for a constant $B$-field, and more generally quantizes the noncommutative geometry defined by the Poisson bivector $\theta=B^{-1}$ on $M=\mathbb{R}^{d}$ for vanishing NS-NS flux $H=\mathrm{d} B=0$.

At the other extreme, consider a constant non-vanishing NS-NS flux $H$ on $M=\mathbb{R}^{d}$ and choose the symmetric gauge $B_{i j}^{\circ}=\frac{1}{3} H_{i j k} x^{k}$ for the Kalb-Ramond field. In this case the worldsheet action (1.5) can be written as

$$
\left.S\right|_{g, \ell_{s}=0}=-\frac{1}{2} \oint_{\Sigma_{2}} \frac{1}{3} H_{i j k} x^{k} \mathrm{~d} x^{i} \wedge \mathrm{d} x^{j}=-\frac{1}{2} \int_{\Sigma_{3}} H_{i j k} \mathrm{~d} x^{i} \wedge \mathrm{d} x^{j} \wedge \mathrm{d} x^{k},
$$

where $\Sigma_{3}$ is a three-dimensional worldvolume with boundary $\partial \Sigma_{3}=\Sigma_{2}$ and we used Stokes' theorem. This is just the well-known Wess-Zumino action, which is needed in particular for a global formulation of the non-linear sigma-model when the target space $M$ is e.g. a torus. The corresponding first order formulation of this action is called the ' $H$-twisted Poisson sigma-model', which captures the topological dynamics of closed strings in a non-trivial $H$-flux background. It is a particular case of a three-dimensional topological field theory called a 'Courant sigma-model', which is defined on a membrane worldvolume.

The somewhat heuristic considerations above can be formalized within the setting of generalized geometry, which provides a higher geometric framework in which to study the geometric 
and non-geometric frames of closed string theory as we have seen in Section 3 together with some aspects of its extension to double field theory.

\subsection{AKSZ theory}

The Alexandrov-Kontsevich-Schwarz-Zaboronsky (AKSZ) construction [3] is a geometric framework for constructing Schwarz-type action functionals in the Batalin-Vilkovisky formalism for $n+1$-dimensional topological sigma-models whose target space is a symplectic Lie $n$-algebroid $E \rightarrow M$. These theories fit into a geometric ladder describing $n$-1-dimensional degrees of freedom in background fields, see e.g. [75] for a review. The open string and membrane sigma-models alluded to above are the first two members of this hierarchy of theories, whose geometric structures we shall now discuss.

Consider first the case $n=1$. A Lie 1-algebroid is simply called a Lie algebroid and consists of a vector bundle $E$ over the target space $M$ that sits in a diagram

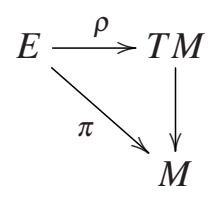

where $\pi$ is the bundle projection and the anchor map $\rho$ to the tangent bundle is compatible with a given Lie bracket $\left[s, s^{\prime}\right]_{E}$ on sections $s, s^{\prime}$ of $E$, in the sense that the following conditions hold:

- $\left[s, f s^{\prime}\right]_{E}=f\left[s, s^{\prime}\right]_{E}+(\rho(s) f) s^{\prime}$,

- $\rho\left(\left[s, s^{\prime}\right]_{E}\right)=\left[\rho(s), \rho\left(s^{\prime}\right)\right]$,

for any function $f \in C^{\infty}(M)$. The first axiom is the Leibniz rule, while the second axiom states that the anchor is a homomorphism between the Lie algebras of sections of $E$ and $T M$. If $M$ is a point, then a Lie algebroid is the same thing as a Lie algebra $\mathfrak{g}$ with zero anchor. On the other hand, the tangent bundle $T M$ of any manifold $M$ is trivially a Lie algebroid with the identity anchor map, and is called the standard Lie algebroid. The notion of a Lie algebroid thus generalizes these two simple examples simultaneously. For our purposes, the most relevant example of a Lie algebroid is the cotangent bundle $E=T^{*} M$ over a Poisson manifold $(M, \theta)$. In this case the anchor is $\rho=\theta^{\sharp}$ and the Lie bracket is the Koszul bracket

$$
[\eta, \xi]_{\mathrm{K}}=£_{\theta^{\sharp} \eta} \xi-£_{\theta^{\sharp} \xi} \eta-\mathrm{d}(\theta(\eta, \xi))
$$

for one-forms $\eta, \xi$ on $M$; in particular, for functions $f, g$ one has $[\mathrm{d} f, \mathrm{~d} g]_{E}=\mathrm{d}\{f, g\}$ where $\{f, g\}=$ $\theta(\mathrm{d} f \wedge \mathrm{d} g)$ is the Poisson bracket induced by $\theta$.

The AKSZ sigma-model in this case is the Poisson sigma-model which is described in more detail below. It quantizes point particles, viewed as boundaries of open strings, in background magnetic fields, with underlying Hamiltonian dynamics governed by the Poisson bracket. This leads to a noncommutative geometry.

Consider next the case $n=2$. A symplectic Lie 2-algebroid is the same thing as a Courant algebroid, which is a vector bundle over $M$ sitting in a diagram like (4.2) with a (not necessarily antisymmetric) bracket $\left[s, s^{\prime}\right]_{E}$ on sections $s, s^{\prime}$ of $E$ and a fibrewise metric $\left\langle s, s^{\prime}\right\rangle_{E}$ satisfying the following conditions: 
- $\left[s,\left[s^{\prime}, s^{\prime \prime}\right]_{E}\right]_{E}=\left[\left[s, s^{\prime}\right]_{E}, s^{\prime \prime}\right]_{E}+\left[s^{\prime},\left[s, s^{\prime \prime}\right]_{E}\right]_{E}$,

- $\left[s, f s^{\prime}\right]_{E}=f\left[s, s^{\prime}\right]_{E}+(\rho(s) f) s^{\prime}$,

- $\rho\left(\left[s, s^{\prime}\right]_{E}\right)=\left[\rho(s), \rho\left(s^{\prime}\right)\right]$,

- $\rho\left(s^{\prime \prime}\right)\left\langle s, s^{\prime}\right\rangle_{E}=\left\langle\left[s^{\prime \prime}, s\right]_{E}, s^{\prime}\right\rangle_{E}+\left\langle s,\left[s^{\prime \prime}, s^{\prime}\right]_{E}\right\rangle_{E}$.

The first two properties endow the vector bundle $E$ with the structure of a 'Leibniz algebroid', and are a generic feature of all symplectic Lie $n$-algebroids; if the bracket is antisymmetric, as in the case $n=1$, then the first axiom is equivalent to the Jacobi identity. If $M$ is a point, then a Courant algebroid is the same thing as a quadratic Lie algebra, i.e. a Lie algebra $\mathfrak{g}$ with an invariant inner product; a natural class of examples is provided by the Drinfeld double $\mathfrak{g} \oplus \mathfrak{g}^{*}$ of a Lie bialgebra $\mathfrak{g}$. For our purposes, the significance of this higher geometric structure is that if the generalized tangent bundle $E=T M \oplus T^{*} M$ is endowed with the Dorfman bracket (3.5), the natural pairing $\left\langle e_{i}, e^{j}\right\rangle=\delta_{i}{ }^{j}$ between $T M$ and $T^{*} M$, and anchor given by the projection $\rho(X+\xi)=X$, then $E$ forms a Courant algebroid called the standard Courant algebroid.

The AKSZ sigma-model in this case is the Courant sigma-model which is studied in detail below. It quantizes closed strings, regarded as boundaries of open membranes, in flux compactifications, with underlying worldsheet Hamiltonian dynamics governed by the Dorfman bracket. This leads to a nonassociative geometry.

The list continues, but the cases with $n \geqslant 3$ are not as well understood (see Section 6 below). Let us now turn to the particular cases of AKSZ sigma-models of direct relevance to us and clarify the statements made above.

\subsection{Poisson sigma-models}

For $n=1$, the most general two-dimensional topological field theory that can be constructed from the AKSZ theory is based on the symplectic Lie algebroid $E=T^{*} M$, and gives rise to the Poisson sigma-model $[74,100]$ defined by the degree zero part of the AKSZ action

$$
S_{\mathrm{AKSZ}}^{(1)}=\int_{\Sigma_{2}}\left(\xi_{i} \wedge \mathrm{d} x^{i}+\frac{1}{2} \theta^{i j}(x) \xi_{i} \wedge \xi_{j}\right)
$$

where $x: \Sigma_{2} \rightarrow M$ are the string fields, $\xi$ are auxiliary one-forms on $\Sigma_{2}$ valued in the cotangent bundle $T^{*} M$, and $\theta=\frac{1}{2} \theta^{i j}(x) \partial_{i} \wedge \partial_{j}$ is a Poisson bivector on $M$. When $\theta=B^{-1}$ is non-degenerate, integrating out the auxiliary one-form fields $\xi_{i}$ yields the topological $B$-field amplitude (1.5). With suitable Dirichlet boundary conditions on the fields, the perturbative expansion of the corresponding path integral leads to the Kontsevich formality maps [80, 41], which we will discuss in Section 5 below. The on-shell condition derived from this action is equivalent to $[\theta, \theta]_{S}=0$, i.e. that the bivector $\theta$ defines a Poisson structure on $M$, but the sigma-model makes sense off-shell as well, for instance when $\theta$ is a twisted Poisson structure.

\subsection{Courant sigma-models}

For $n=2$, we take the symplectic Lie 2-algebroid to be the standard Courant algebroid $E=$ $T M \oplus T^{*} M$, and define the three-dimensional topological field theory on a membrane worldvolume 
$\Sigma_{3}$ by the degree zero part of the AKSZ action

$$
S_{\mathrm{AKSZ}}^{(2)}=\int_{\Sigma_{3}}\left(\phi_{i} \wedge \mathrm{d} x^{i}+\frac{1}{2} \eta_{I J} \alpha^{I} \wedge \mathrm{d} \alpha^{J}-\rho_{I}^{i} \phi_{i} \wedge \alpha^{I}+\frac{1}{6} T_{I J K}(x) \alpha^{I} \wedge \alpha^{J} \wedge \alpha^{K}\right),
$$

where $x: \Sigma_{3} \rightarrow M$ are the membrane fields, $\alpha$ are one-forms on $\Sigma_{3}$ valued in $E$, and $\phi$ are auxiliary two-forms on $\Sigma_{3}$ valued in the cotangent bundle $T^{*} M$. The fibre metric has components $\eta_{I J}=$ $\left\langle s_{I}, s_{J}\right\rangle_{E}$ in a local basis of sections $s_{I}$ of $E$, the anchor map has components $\rho\left(s_{I}\right)=\rho_{I}{ }^{i} e_{i}$, and the three-form $T_{I J K}(x)=\left\langle s_{I},\left[s_{J}, s_{K}\right]_{E}\right\rangle_{E}$ can accomodate all four geometric and non-geometric fluxes in the T-duality orbit (3.1). This is called the Courant sigma-model [96].

Let us begin by describing the geometric $H$-flux frame. We write the one-forms as $\alpha=\left(\alpha^{I}\right)=$ $\left(\alpha^{i}, \xi_{i}\right)$ corresponding to the splitting $E=T M \oplus T^{*} M$ and use the $H$-twisted Dorfman bracket from (3.6) with vanishing torsion to write the open membrane action

$$
S_{H}^{(2)}=\int_{\Sigma_{3}}\left(\phi_{i} \wedge \mathrm{d} x^{i}+\alpha^{i} \wedge \mathrm{d} \xi_{i}-\phi_{i} \wedge \alpha^{i}+\frac{1}{6} H_{i j k}(x) \alpha^{i} \wedge \alpha^{j} \wedge \alpha^{k}\right) .
$$

With suitable Dirichlet boundary conditions on the fields, by integrating out the auxiliary two-form fields $\phi_{i}$ we arrive at the action on the closed string worldsheet $\Sigma_{2}=\partial \Sigma_{3}$ given by

$$
S_{H}^{(2)}=\oint_{\Sigma_{2}} \xi_{i} \wedge \mathrm{d} x^{i}+\int_{\Sigma_{3}} \frac{1}{6} H_{i j k}(x) \mathrm{d} x^{i} \wedge \mathrm{d} x^{j} \wedge \mathrm{d} x^{k} .
$$

This is just the Wess-Zumino action, which for constant $H$-flux falls entirely on the worldsheet $\Sigma_{2}$ as in (4.1). One can also add a boundary perturbation to the membrane sigma-model by an arbitrary bivector $\theta$ on $M$ of the form given in (4.4). This defines the action of the $H$-twisted Poisson sigmamodel [78], whose on-shell conditions imply $[\theta, \theta]_{S}=\Lambda^{3} \theta^{\sharp} H$, so that $\theta$ defines an $H$-twisted Poisson structure on the target space $M$; in particular, the Jacobi identity for the corresponding bracket defined by $\theta$ is violated by the NS-NS three-form flux.

Now let us consider the locally non-geometric $R$-flux frame. Using the $R$-twisted Dorfman bracket from (3.7) with vanishing torsion and $Q$-flux, and with notation as above, after integrating out the auxiliary fields $\phi_{i}$ again we arrive at the open membrane sigma-model with (rescaled) $R$-flux given by the action

$$
S_{R}^{(2)}=\int_{\Sigma_{3}}\left(\mathrm{~d} \xi_{i} \wedge \mathrm{d} x^{i}+\frac{\ell_{s}^{4}}{18 \hbar^{2}} R^{i j k}(x) \xi_{i} \wedge \xi_{j} \wedge \xi_{k}\right) .
$$

When the $R$-flux is constant, with suitable Dirichlet boundary conditions the equations of motion for the boundary string fields $x^{i}$ imply $\xi_{i}=\mathrm{d} p_{i}$ for local fields $p$ valued in the fibres of the cotangent bundle $T^{*} M$ (up to harmonic forms on $\Sigma_{2}$ ), so that using Stokes' theorem and linearizing the resulting action with auxiliary one-form fields $\eta_{I}$ valued in $E$ leads to the boundary action [89]

$$
S_{R}^{(2)}=\oint_{\Sigma_{2}}\left(\eta_{I} \wedge \mathrm{d} x^{I}+\frac{1}{2} \Theta^{I J} \eta_{I} \wedge \eta_{J}\right)
$$

Here $x=\left(x^{I}\right)=\left(x^{i}, p_{i}\right): \Sigma_{2} \rightarrow T^{*} M$ are string fields valued in the cotangent bundle of $M$, so that the effective target space is now the phase space. This action defines a Poisson sigma-model for the bivector

$$
\Theta=\left(\Theta^{I J}\right)=\left(\begin{array}{cc}
R(p) & 1_{d} \\
-1_{d} & 0
\end{array}\right) \quad \text { with } \quad R(p)^{i j}=\frac{\ell_{s}^{4}}{3 \hbar^{2}} R^{i j k} p_{k}
$$


The corresponding quantum phase space brackets

$$
\left[x^{I}, x^{J}\right]=\mathrm{i} \hbar \Theta^{I J}(x)
$$

coincide precisely with the $R$-space commutation relations (3.27). Note that for $d=3$ this twisted Poisson structure is formally identical to (2.4) with a linear magnetic field (2.15) under the magnetic duality transformation (3.28), and in particular the commutators (4.11) together with the corresponding Jacobiators

$$
\left[x^{I}, x^{J}, x^{K}\right]=-\hbar^{2}[\Theta, \Theta]_{\mathrm{S}}^{I J K}=\left(\begin{array}{cc}
\ell_{S}^{4} R^{i j k} & 0 \\
0 & 0
\end{array}\right)
$$

yield a noncommutative and nonassociative phase space geometry.

One important caveat with this derivation is that the setting of the $Q$-flux to zero in (3.7) does not define a Courant algebroid structure, as is evident from (3.8). This simply reflects the fact that the nonassociative geometry of the $R$-flux frame violates the strong constraint of double field theory, as mentioned previously, so that the corresponding membrane sigma-model does not define a Courant sigma-model. It can, however, be obtained in a precise way via projection from a proper Courant sigma-model defined on the doubled space of double field theory that incorporates all fluxes of (3.1) in a manifestly T-duality invariant way [44], which clarifies precisely the geometric algebroid structure underlying the non-geometric frames of closed string theory, and also how gauge invariance is restored in the non-geometric membrane sigma-models through the Bianchi identities among the fluxes of the T-duality chain (3.1). In [44] it is also shown how to treat the noncommutative geometry of the globally non-geometric $Q$-flux frame in an analogous manner, and how to generally treat the non-geometry through Courant sigma-models via the open-closed string reparameterization (1.9) of the algebroid structure maps, thereby clarifying the evident similarities we have seen between the phase space and double field theory frameworks for non-geometry (see also [58, 59]).

\section{Quantization of non-geometric backgrounds}

In this section we will describe the quantization of the twisted Poisson structure (4.10) describing the nonassociative geometry of the closed string phase space in the $R$-flux frame, and then present some of its far reaching applications to non-geometric string theory.

\subsection{Quantization of topological string theory}

Suitable functional integrals in the $R$-flux Poisson sigma-model (4.9) reproduce Kontsevich's formality maps for global deformation quantization of twisted Poisson manifolds [80]. The formality maps $U_{n}$ take $n$ multivector fields $\mathscr{X}_{1}, \ldots, \mathscr{X}_{n}$ of degrees $k_{1}, \ldots, k_{n}$ to multidifferential operators $D_{\Gamma}\left(\mathscr{X}_{1}, \ldots, \mathscr{X}_{n}\right)$ of degree $2-2 n+k_{1}+\cdots+k_{n}$. They have a combinatorial expansion as a sum over graphs

$$
U_{n}\left(\mathscr{X}_{1}, \ldots, \mathscr{X}_{n}\right)=\sum_{\Gamma_{n} \in G_{n}} w_{\Gamma_{n}} D_{\Gamma_{n}}\left(\mathscr{X}_{1}, \ldots, \mathscr{X}_{n}\right)
$$


where $G_{n}$ is the set of admissible graphs $\Gamma_{n}$, with $n$ vertices and edges $\left\{e_{i}^{1}, \ldots, e_{i}^{k_{i}}\right\}_{i=1}^{n}$, which can be drawn in the configuration space $\mathbb{H}_{n}$ of $n$ points on the hyperbolic plane with prescribed weights

$$
w_{\Gamma_{n}}=\frac{1}{(2 \pi)^{\sum_{i} k_{i}}} \int_{\mathbb{H}_{n}} \bigwedge_{i=1}^{n}\left(\mathrm{~d} \phi_{e_{i}^{1}} \wedge \cdots \wedge \mathrm{d} \phi_{e_{i}^{k_{i}}}\right) .
$$

In particular, inserting any bivector $\mathscr{X}_{i}=\Theta=\frac{1}{2} \Theta^{I J} \partial_{I} \wedge \partial_{J}$ into all slots gives a sum of bidifferential operators, which can be represented diagrammatically by graphs with edges emanating along the legs of $\Theta$ and operating on functions $f, g$ sitting on the boundary of the hyperbolic plane. This defines the star product

$$
f \star g=\sum_{n=0}^{\infty} \frac{(\mathrm{i} \hbar)^{n}}{n !} U_{n}(\Theta, \ldots, \Theta)(f, g)=: \mathscr{P}(\Theta)(f, g),
$$

which up to order $\hbar^{2}$ is given explicitly by

$$
\begin{aligned}
f \star g=f \cdot g+ & \frac{\mathrm{i} \hbar}{2} \Theta^{I J} \partial_{I} f \cdot \partial_{J} g-\frac{\hbar^{2}}{4} \Theta^{I J} \Theta^{K L} \partial_{I} \partial_{K} f \cdot \partial_{J} \partial_{L} g \\
& -\frac{\hbar^{2}}{6} \Theta^{I J} \partial_{J} \Theta^{K L}\left(\partial_{I} \partial_{K} f \cdot \partial_{L} g-\partial_{K} f \cdot \partial_{I} \partial_{L} g\right)+O\left(\hbar^{3}\right) .
\end{aligned}
$$

The order $\hbar$ term is the semiclassical contribution which is proportional to the classical bracket $\{f, g\}=\Theta(\mathrm{d} f \wedge \mathrm{d} g)$ defined by the bivector $\Theta$.

As a simple example, let us consider a constant bivector $\theta$ in the Poisson sigma-model (4.4). The basic graph $\Gamma_{1}$ with a single vertex and two edges contributes the weight

$$
w_{\Gamma_{1}}=\frac{1}{(2 \pi)^{2}} \int_{0}^{2 \pi} \mathrm{d} \psi \int_{0}^{\psi} \mathrm{d} \phi=\frac{1}{(2 \pi)^{2}}\left[\frac{1}{2} \psi^{2}\right]_{0}^{2 \pi}=\frac{1}{2} .
$$

In this case all graphs and hence weight integrals (5.2) factorize in terms of this basic graph $\Gamma_{1}$, so that the sum (5.1) truncates to

$$
U_{n}(\theta, \ldots, \theta)=\left(\frac{1}{2}\right)^{n} \theta^{i_{1} j_{1}} \cdots \theta^{i_{n} j_{n}}\left(\partial_{i_{1}} \cdots \partial_{i_{n}}\right) \otimes\left(\partial_{j_{1}} \cdots \partial_{j_{n}}\right) .
$$

The star product (5.3) thus becomes

$$
f \star g=\sum_{n=0}^{\infty} \frac{(\mathrm{i} \hbar)^{n}}{n !}\left(\frac{1}{2}\right)^{n} \theta^{i_{1} j_{1}} \cdots \theta^{i_{n} j_{n}} \partial_{i_{1}} \cdots \partial_{i_{n}} f \cdot \partial_{j_{1}} \cdots \partial_{j_{n}} g,
$$

which is just the Moyal-Weyl star product (1.13).

In general, if $\Theta$ is not constant but still satisfies $\Theta^{I J} \partial_{J} \Theta^{K L}=0$, then the series (5.3) again exponentiates exactly as in the case of a constant bivector which led to the Moyal-Weyl star product. This is the case for the bivector (4.10) with constant $R$-flux, leading to the star product [89]

$$
f \star g=\cdot \exp \left(\frac{\mathrm{i} \hbar}{2}\left[\frac{\ell_{s}^{4}}{3 \hbar^{2}} R^{i j k} p_{k} \partial_{i} \otimes \partial_{j}+\partial_{i} \otimes \tilde{\partial}^{i}-\tilde{\partial}^{i} \otimes \partial_{i}\right]\right)(f \otimes g),
$$

where $\tilde{\partial}^{i}=\frac{\partial}{\partial p_{i}}$ denote momentum derivatives; in Fourier space it reads as

$$
(f \star g)(x, p)=\int \mathrm{d} k \mathrm{~d} \tilde{k} \int \mathrm{d} q \mathrm{~d} \tilde{q} \tilde{f}(k, \tilde{k}) \tilde{g}(q, \tilde{q}) \mathrm{e}^{\frac{\mathrm{i} \hbar}{2}\left(\tilde{k}^{i} q_{i}-k_{i} \tilde{q}^{i}\right)} \mathrm{e}^{-\frac{\mathrm{i} k_{s}^{4}}{6 \hbar} R^{i j k} k_{i} q_{j} p_{k}} \mathrm{e}^{\mathrm{i}(k+q)_{i} x^{i}} .
$$


This is a deformation by $R$-flux of the usual phase space Moyal product for deformation quantization in ordinary quantum mechanics, see e.g. [118] for a concise review. Other approaches to deriving this star product can be found in [89, 16, 90, 83]. Various formal properties of this and other classes of nonassociative star products in deformation quantization are discussed in $[37,116]$.

The formality maps $U_{n}$ define quasi-isomorphisms between differential graded $L_{\infty}$-algebras, relating Schouten brackets $[,]_{S}$, i.e. the obvious extensions of the Lie bracket of vector fields to multivector fields, to Gerstenhaber brackets $[\text {, }]_{\mathrm{G}}$, i.e. the obvious extensions of the commutator of differential operators to multidifferential operators. As such they satisfy a set of 'formality conditions'. In particular, one of these conditions reads as

$$
\mathrm{i} \hbar \mathscr{P}\left([\Theta, \Theta]_{\mathrm{S}}\right)=[\mathscr{P}(\Theta), \star]_{\mathrm{G}} .
$$

This quantifies nonassociativity, since when applied to triples of functions $f, g, h$, it follows that $[\Theta, \Theta]_{S} \neq 0$ if and only if

$$
(f \star g) \star h-f \star(g \star h) \neq 0 .
$$

That is, $\Theta$ is a Poisson bivector if and only if the star product is associative. For the $R$-space with constant locally non-geometric flux, the star commutators

$$
\left[x^{I}, x^{J}\right]_{\star}:=x^{I} \star x^{J}-x^{J} \star x^{I}=\mathrm{i} \hbar \Theta^{I J}(x)
$$

have the corresponding non-vanishing star Jacobiators

$$
\left[x^{i}, x^{j}, x^{k}\right]_{\star}=\ell_{s}^{4} R^{i j k} .
$$

For general phase space functions, the nonassociativity of the star product can be expressed in closed form as

$$
(f \star g) \star h=\varphi_{f, g, h}(f \star(g \star h)):=\star \exp \left(\frac{\ell_{s}^{4}}{6} R^{i j k} \partial_{i} \otimes \partial_{j} \otimes \partial_{k}\right)(f \otimes(g \otimes h)),
$$

where the associators

$$
f \star(g \star h) \stackrel{\varphi_{f, g, h}}{\longrightarrow}(f \star g) \star h
$$

satisfy pentagon relations implied by the (higher) formality conditions [89], which state that the diagram

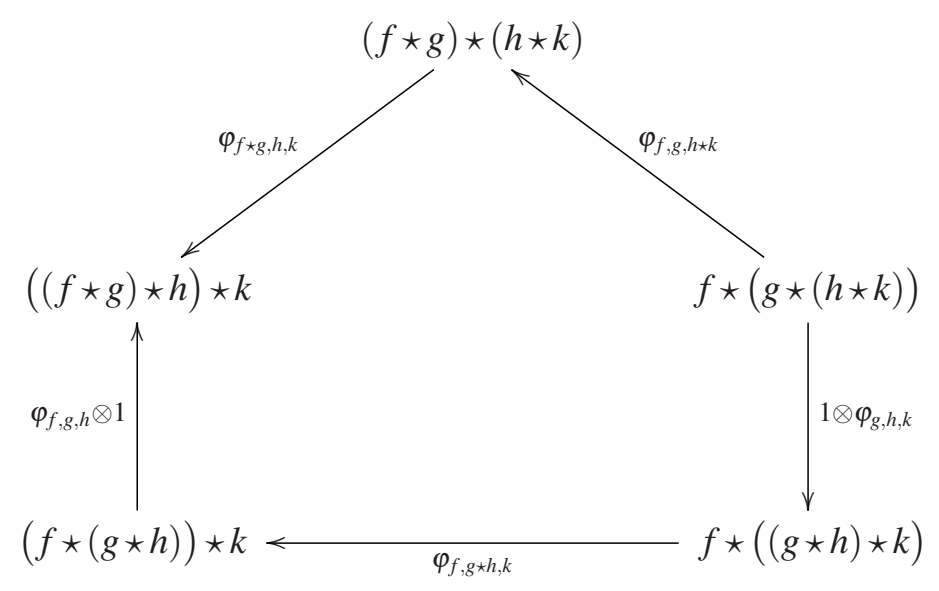


commutes for all functions $f, g, h, k$. In particular, the translation group three-cocycle discussed in Section 2 is realised here through a nonassociative version of the Baker-Campbell-Hausdorff formula which yields

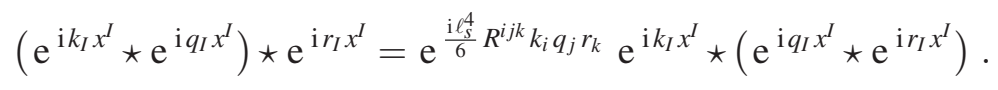

In the remainder of this section we consider various applications of this nonassociative phase space star product formalism.

\subsection{Triproducts}

We shall now address Question (Q2) from Section 1. Given $n$ functions $f_{1}, \ldots, f_{n} \in C^{\infty}(M)$ on the target space $M$, using the nonassociative phase space star product we define the triproducts

$$
f_{1} \triangle f_{2} \triangle \cdots \triangle f_{n}:=\lim _{p \rightarrow 0} f_{1} \star\left(f_{2} \star\left(\cdots\left(f_{n-1} \star f_{n}\right) \cdots\right)\right),
$$

where we have chosen a particular bracketing for the star products of functions. It was shown by [11] that the result of this operation can be expressed in terms of a tridifferential operator as

$$
f_{1} \triangle f_{2} \triangle \cdots \triangle f_{n}=\cdot \exp \left(-\frac{\ell_{s}^{4}}{6} \sum_{1 \leqslant a<b<c \leqslant n} R^{i j k} \partial_{i}^{a} \otimes \partial_{j}^{b} \otimes \partial_{k}^{c}\right)\left(f_{1} \otimes f_{2} \otimes \cdots \otimes f_{n}\right),
$$

where $\partial_{i}^{a}$ denotes the action of the derivative $\partial_{i}$ in the $a$-th factor of the tensor product $f_{1} \otimes f_{2} \otimes$ $\cdots \otimes f_{n}$. This remarkable algebraic structure has the following properties.

Firstly, it agrees with the triproducts of tachyon vertex operators after T-duality in worldsheet perturbation theory around flat space with constant $H$-flux, as we discussed earlier. This formula was derived to linear order in the $R$-flux in [33], where its all orders exponential form was conjectured, and subsequently extrapolated to non-constant fluxes appropriate to curved backgrounds $M$ in [34]. Here we find an explicit calculational derivation of these conjectural expressions from the nonassociative phase space star product, which lends further credibility to the purported description of the locally non-geometric flux background.

Secondly, since the momentum is set to $p=0$, for $n=2$ there is no deformation of the usual pointwise product of fields: $f \triangle g=f \cdot g$, as anticipated from the closed string theory considerations of Section 1. For $n=3$ the triproduct was originally introduced in [115] as a proposal for the quantization of Nambu brackets [92], which we will discuss in more detail later on. It quantizes the tribracket defined by (1.17), and in particular one has

$$
\left[x^{i}, x^{j}, x^{k}\right]_{\triangle}=\ell_{s}^{4} R^{i j k},
$$

which agrees with the Jacobiator (5.13).

Thirdly, the triproducts exhibit on-shell associativity:

$$
\int \mathrm{d} x f_{1} \triangle \cdots \triangle f_{n}=\int \mathrm{d} x f_{1} \cdots f_{n},
$$

which follows from on-shell momentum conservation [11]. As we discussed earlier, this is expected and in fact necessary from the string theory perspective: As on-shell closed string theory 
is described a two-dimensional quantum field theory, it is described by an associative operator algebra. In particular, the on-shell associativity agrees with the crossing symmetry of $n$-point correlation functions on the sphere $S^{2}$.

Finally, as also mentioned before, the triproducts violate the strong constraint of double field theory. Recalling the microscopic origin of the $R$-flux in terms of a bivector from (3.19), the expansion of the triproduct for $n=3$ reads as

$$
f \triangle g \triangle h=f \cdot g \cdot h-\frac{\ell_{s}^{4}}{6} \tilde{\partial}^{[i} \tilde{\theta}^{j k]} \partial_{i} f \cdot \partial_{j} g \cdot \partial_{k} h+O\left(\ell_{s}^{8}\right),
$$

and the corrections to the pointwise product of fields vanishes by the strong constraint. Thus the nonassociative geometry of the closed string background is only probed if the strong constraint between the background and fluctuations is weakened, a point which is presently under debate.

\subsection{Nonassociative quantum mechanics}

Let us now investigate some of the physical consequences of the nonassociative deformation of quantum mechanics in the $R$-flux background. The standard operator-state formulation of quantum mechanics cannot handle nonassociative structures: Operators which act on a Hilbert space necessarily associate. However, we can generalize the phase space formulation of quantum mechanics [118] to provide a completely quantitative and physically viable formulation of nonassociative quantum mechanics [90]. Other approaches to nonassociative quantum mechanics based on the magnetic monopole algebra (2.3) are found in [36, 35].

The idea behind phase space quantum mechanics is to treat position and momentum variables on equal footing. In this setting generic 'operators' become complex-valued functions on phase space, with the operator product provided by the star product and traces given by integration of functions; an 'observable' is then a real-valued function on phase space. Dynamics of observables $A$ are governed by Heisenberg-type time evolution equations

$$
\dot{A}=\frac{\mathrm{i}}{\hbar}[H, A]_{\star},
$$

for a given classical Hamiltonian $H$.

The key properties of the nonassociative phase space star product (5.8) that are needed in this description are as follows. Firstly, since the star product $f \star g$ again differs from $f \cdot g$ by a total derivative, and likewise $f \star(g \star h)$ differs from $(f \star g) \star h$ by a total derivative, one again has the 2-cyclicity and 3-cyclicity properties (1.14) and (1.15) that we encountered for the Moyal-Weyl product. Recall that these features were crucial for compatibility of the star product formalism with the axioms of conformal field theory; in the present context, they refer to the fact that the star product leads to a traceless commutator and associator. However, there are in general inequivalent quartic expressions, for example

$$
\int \mathrm{d} x f \star(g \star(h \star k)) \neq \int \mathrm{d} x f \star((g \star h) \star k),
$$

see $[90,88]$ for a detailed discussion. Using 2-cyclicity we deduce the crucial positivity property

$$
\int \mathrm{d} x \bar{f} \star f \geqslant 0 .
$$


Finally, the nonassociative star product is Hermitian, in the sense that $\overline{f \star g}=\bar{g} \star \bar{f}$, and unital, in the sense that the constant unit function 1 is still an identity element for the nonassociative star product algebra: $f \star 1=f=1 \star f$. All of these properties are completely analogous to those of the usual Moyal product in canonical phase space quantum mechanics and they mimick the expected features of the operator product; indeed, the star product (5.8) for the constant $R$-flux background is the simplest example of a nonassociative star product and provides the natural extension of the Moyal-Weyl star product (1.13) to the non-geometric string background.

A 'state' in nonassociative quantum mechanics is determined by a set of square-integrable phase space wavefunctions $\psi_{a}$ which are normalized:

$$
\int \mathrm{d} x\left|\psi_{a}\right|^{2}=1
$$

and a collection of statistical probabilities $\mu_{a} \in[0,1]$ obeying

$$
\sum_{a} \mu_{a}=1
$$

The 'expectation value' of an operator $A$ is then defined by

$$
\langle A\rangle_{\psi}=\sum_{a} \mu_{a} \int \mathrm{d} x \overline{\psi_{a}} \star\left(A \star \psi_{a}\right)=\int \mathrm{d} x W_{\psi} \cdot A,
$$

where we introduced the state function

$$
W_{\psi}=\sum_{a} \mu_{a} \psi_{a} \star \overline{\psi_{a}}
$$

which is the analogue of the Wigner distribution function; in particular, it is real-valued and normalized: $\langle 1\rangle_{\psi}=\int \mathrm{d} x W_{\psi}=1$. However, even in canonical phase space quantum mechanics [118], one of the pitfalls of the formalism is that state functions are not necessarily non-negative and so only determine quasi-probability distribution functions in general.

As a simple example to familiarize ourselves with the phase space formulation of quantum mechanics, let us consider the simplest case of the free particle with Hamiltonian

$$
H(x, p)=\sum_{i=1}^{d} \frac{p_{i}^{2}}{2 m}
$$

and vanishing $R$-flux. We denote the corresponding Moyal star product from (5.8) with $R^{i j k}=0$ by $\star_{0}$ and look for solutions of the time-independent Schrödinger equation

$$
H \star_{0} W_{\psi}=E W_{\psi} .
$$

This can be expressed as a second order partial differential equation

$$
\frac{1}{2 m} \sum_{i=1}^{d}\left(p_{i}^{2}+\mathrm{i} \hbar \partial_{i}-\frac{\hbar^{2}}{4} \partial_{i}^{2}\right) W_{\psi}=E W_{\psi}
$$

for the real-valued Wigner distribution function $W_{\psi}(x, p)$. It collapses to a pair of partial differential equations, its real and imaginary parts. The imaginary part

$$
\sum_{i=1}^{d} p_{i} \partial_{i} W_{\psi}(x, p)=0
$$


restricts $W_{\psi}(x, p)=W_{\psi}(p)$ to be independent of $x$. The real part

$$
\sum_{i=1}^{d}\left(p_{i}^{2}-\frac{\hbar^{2}}{4} \partial_{i}^{2}-2 m E\right) W_{\psi}(p)=0
$$

is satisfied for arbitrary real functions $W_{\psi}(p)$ of momentum with the energy eigenvalues

$$
E=E_{p}=\sum_{i=1}^{d} \frac{p_{i}^{2}}{2 m} .
$$

This calculation can be extended to include an anisotropic harmonic oscillator potential of frequencies $\omega_{i}$, for which the real part of the second order partial differential equation can be separated into Laguerre equations in the phase space variables $z_{i}=\frac{2}{m \hbar}\left(m \omega_{i}^{2} x_{i}^{2}+p_{i}^{2}\right)$ for $i=1, \ldots, d$ [118]. The corresponding solutions in terms of Laguerre polynomials reproduce the anticipated harmonic oscillator spectrum

$$
E=E_{n}=\sum_{i=1}^{d} \hbar \omega_{i}\left(n_{i}+\frac{1}{2}\right) \quad \text { with } \quad n=\left(n_{i}\right) \in \mathbb{N}_{0}^{d}
$$

in $d$ dimensions. The extension of these considerations to a free particle moving in a non-vanishing $R$-flux background is discussed in [114], and from the alternative associative framework of symplectic realisation of the twisted Poisson structure (4.10) in [82].

The operator-state correspondence can be described in this framework as follows. The idea is that operators should still be combined together with an associative operation, since nonassociativity should not affect correlation functions. To this end we introduce two conjugate "operator algebras" by defining left and right compositions of observables $A, B$ as

$$
(A \circ B) \star f:=A \star(B \star f) \quad \text { and } \quad f \star(A \bar{\circ} B):=(f \star A) \star B,
$$

for a test function $f$. By definition the composition products are associative, since

$$
\left(A_{1} \circ A_{2} \circ \cdots \circ A_{n}\right) \star f=A_{1} \star\left(A_{2} \star \cdots \star\left(A_{n} \star f\right) \cdots\right) .
$$

They are furthermore unital: $A \circ 1=A=1 \circ A$, and the composition products of basic coordinate monomials coincide with their star products, e.g. $x^{i} \circ x^{i}=x^{i} \star x^{i}=\left(x^{i}\right)^{2}$ and $p_{i} \circ p_{i}=p_{i} \star p_{i}=\left(p_{i}\right)^{2}$. However, in general the composition product $A \circ B$ of two functions $A$ and $B$ is not a function, but rather a differential operator; the composition products arise as the embedding of the nonassociative star product algebra of functions as a subspace (but not as a subalgebra) of an associative algebra of differential operators on phase space. In the conventional frameworks where $\star$ is associative, the composition product and the star product coincide. The physical and geometric meaning of the associative composition algebra of differential operators is elucidated by [82] from the perspective of symplectic realisation of the twisted Poisson structure (4.10).

We make the convention that the conjugate composition product $\bar{o}$ is always evaluated before ○. Then a 'state' $\rho_{\psi}$ is an expression of the form

$$
\rho_{\psi}=\sum_{a} \mu_{a} \psi_{a} \bar{\circ} \overline{\psi_{a}} .
$$


This quantity should be thought of as a special representation of a "density matrix", which in the associative setting would be the same as the Wigner distribution function; in particular, the expectation values (5.28) of operators are determined via traces with $\rho_{\psi}$ through

$$
\langle A\rangle_{\psi}=\int \mathrm{d} x A \star \rho_{\psi} .
$$

The expectation values of compositions of operators are then given by

$$
\begin{aligned}
\left\langle A_{1} \circ A_{2} \circ \cdots \circ A_{n}\right\rangle_{\psi} & =\int \mathrm{d} x\left(A_{1} \circ A_{2} \circ \cdots \circ A_{n}\right) \star \rho_{\psi} \\
& =\sum_{a} \mu_{a} \int \mathrm{d} x\left(A_{1} \star\left(A_{2} \star \cdots \star\left(A_{n} \star \psi_{a}\right) \cdots\right)\right) \star \overline{\psi_{a}} .
\end{aligned}
$$

Let us make some simple consistency checks of this purported nonassociative version of quantum mechanics. First, let us check reality. A straightforward calculation using the above definitions and the properties of the nonassociative star product gives

$$
\overline{\langle A\rangle_{\psi}}=\sum_{a} \mu_{a} \int \mathrm{d} x \overline{\left(A \star \psi_{a}\right)} \star \psi_{a}=\sum_{a} \mu_{a} \int \mathrm{d} x \overline{\psi_{a}} \star\left(\bar{A} \star \psi_{a}\right)=\langle\bar{A}\rangle_{\psi}
$$

It follows that the expectation values of observables, i.e. real functions, are real. Similarly, one checks positivity: $\langle\bar{A} \circ A\rangle_{\psi} \geqslant 0$. These derivations are carried out in complete analogy with the corresponding calculations in canonical phase space quantum mechanics; however, in the nonassociative case an extra line or two is always required in the computation.

Next we check that observables, i.e. real functions $A=\bar{A}$, have real eigenvalues. For this, consider the "star-genvalue equation" $A \star f=\lambda f$ for a complex number $\lambda$; complex conjugation of this equation using Hermiticity of the star product yields $\bar{f} \star \bar{A}=\bar{\lambda} \bar{f}$. From this we calculate

$$
\bar{f} \star(A \star f)-(\bar{f} \star A) \star f=(\lambda-\bar{\lambda})(\bar{f} \star f) .
$$

In the associative case this would immediately imply that $\lambda=\bar{\lambda}$ is real, since the left-hand side would automatically vanish. In the nonassociative case this is not generally true, but using 3cyclicity and 2-cyclicity we can integrate both sides this equation to get

$$
0=(\lambda-\bar{\lambda}) \int \mathrm{d} x|f|^{2}
$$

Since the integral on the right-hand side is non-zero for $f \neq 0$, it follows that $\lambda=\bar{\lambda}$. A similar calculation establishes that eigenfunctions with different eigenvalues are orthogonal in the $L^{2}$-inner product.

\subsection{Spacetime quantization}

As a concrete application of the formalism, we will now show how nonassociative quantum mechanics in the $R$-flux background leads to a coarse-graining of spacetime. There are several ways in which to see this. In standard quantum mechanics it is a fundamental result that pairs of noncommuting operators cannot have simultaneous eigenvalues. Similarly, in the present case we can show that triples of nonassociating operators cannot have simultaneous eigenvalues. Suppose that 
$W_{\psi}$ is a state function which simultaneously diagonalizes a triple of basic phase space coordinate operators: $x^{I} \star W_{\psi}=\lambda^{I} W_{\psi}, x^{J} \star W_{\psi}=\lambda^{J} W_{\psi}$ and $x^{K} \star W_{\psi}=\lambda^{K} W_{\psi}$ for eigenvalues $\lambda^{I}, \lambda^{J}, \lambda^{K} \in \mathbb{R}$. By repeatedly applying the cyclicity properties of the star product we compute

$$
\begin{aligned}
\int \mathrm{d} x\left(\left(x^{I} \star x^{J}\right) \star x^{K}\right) \star W_{\psi} & =\int \mathrm{d} x\left(x^{I} \star x^{J}\right) \star\left(x^{K} \star W_{\psi}\right) \\
& =\lambda^{K} \int \mathrm{d} x\left(x^{I} \star x^{J}\right) \star W_{\psi} \\
& =\lambda^{K} \int \mathrm{d} x x^{I} \star\left(x^{J} \star W_{\psi}\right) \\
& =\lambda^{K} \lambda^{J} \lambda^{I}
\end{aligned}
$$

and similarly

$$
\int \mathrm{d} x\left(x^{I} \star\left(x^{J} \star x^{K}\right)\right) \star W_{\psi}=\lambda^{I} \lambda^{K} \lambda^{J}
$$

Taking the difference of these two equations implies the vanishing Jacobiators $\left[x^{I}, x^{J}, x^{K}\right]_{\star}=0$, which contradicts the basic nonassociative deformation provided by the position coordinate operators in (5.13). This implies a coarse-graining of spacetime by the non-geometric $R$-flux background.

To compute the coarse-graining quantitatively, we introduce oriented area and volume uncertainty operators

$$
\begin{aligned}
\mathrm{A}^{I J} & =\operatorname{Im}\left(\left[\widetilde{x}^{I}, \widetilde{x}^{J}\right]_{\star}\right)=-\mathrm{i}\left(\widetilde{x}^{I} \star \widetilde{x}^{J}-\widetilde{x}^{J} \star \widetilde{x}^{I}\right), \\
\mathrm{V}^{I J K} & =\frac{1}{3} \operatorname{Re}\left(\widetilde{x}^{I} \star\left[\widetilde{x}^{J}, \widetilde{x}^{K}\right]_{\star}+\widetilde{x}^{K} \star\left[\widetilde{x}^{I}, \widetilde{x}^{J}\right]_{\star}+\widetilde{x}^{J} \star\left[\widetilde{x}^{K}, \widetilde{x}^{I}\right]_{\star}\right),
\end{aligned}
$$

where we introduced the shifted coordinates $\widetilde{x}^{I}:=x^{I}-\left\langle x^{I}\right\rangle_{\psi}$ appropriate to uncertainties in measurements. These definitions mimick the vector product and triple scalar product of vectors in the respective coordinate directions of phase space, and their expectation values measure the minimal area and volume uncertainties between corresponding operators. A simple computation using (5.12), (5.13) and the integration properties above leads to the non-vanishing expectation values

$$
\left\langle\mathrm{A}^{x^{i}, p_{j}}\right\rangle_{\psi}=\hbar \delta^{i}{ }_{j}, \quad\left\langle\mathrm{~A}^{i j}\right\rangle_{\psi}=\frac{\ell_{s}^{4}}{3 \hbar} R^{i j k}\left\langle p_{k}\right\rangle_{\psi} \quad \text { and } \quad\left\langle\mathrm{V}^{i j k}\right\rangle_{\psi}=\frac{1}{2} \ell_{s}^{4} R^{i j k} .
$$

The first expectation value gives the usual Planck cells of canonical quantum phase space with the Planck quantum of minimal area $\hbar$, while the second expectation value is a new uncertainty measurement giving minimal spacetime areas in the directions transverse to the motion of closed strings (as originally conjectured by [84]). The third expectation value is the most interesting: It implies a quantized spacetime with a quantum of minimal volume $\frac{1}{2} \ell_{s}^{4} R^{i j k}$.

This coarse-graining of spacetime has the following physical interpretation from the perspective of non-geometric string theory, which corroborates the argument that there can be no D0-branes in the locally non-geometric background [117]. In $d=3$ dimensions, applying a triple T-duality $\mathscr{T}_{(123)}$ maps the $R$-space to the three-torus with $H$-flux and a D0-brane to a D3-brane wrapping the torus. However, this latter configuration is not allowed as it suffers from the Freed-Witten anomaly [56], i.e. it violates the Bianchi identity $\mathrm{d} F=H$ for the gauge flux $F$ on a D3-brane. 
Generally, Freed-Witten anomaly cancellation would require that the degree three integer cohomology class of the NS-NS flux $H$ be equal to the torsion characteristic class which measures the obstruction to a $\operatorname{spin}^{c}$ structure on the target space $M$. But if $M$ is a torus, then its cohomology is torsion-free and it is a $\operatorname{spin}^{c}$ manifold, so that non-vanishing $H$-flux is not allowed. In the T-dual frame, this implies that placing a point-like object in the $R$-flux background is not allowed. This is yet another manifestation of the local non-geometry of the $R$-flux background, which we have reproduced here in a completely quantitative way through our formalism of nonassociative quantum mechanics.

\subsection{Quantization of Nambu brackets}

As another application of the nonassociative phase space deformation quantization, let us examine the problem of quantizing Nambu-Poisson structures, which have notably appeared in recent years in effective theories of M2-branes and M5-branes in M-theory, see e.g. [67] for a review. Nambu mechanics involves multi-Hamiltonian dynamics with generalized Poisson brackets $\{f, g, h\}$ of functions obeying the Leibniz rule and a "fundamental identity" which is a higher generalization of the Jacobi identity for Lie brackets [92]. Nambu used this algebraic structure to reformulate the Euler equations describing the dynamics of a rotating rigid body in $\mathbb{R}^{3}$ in the absence of applied torques, in the hope of obtaining new generalized integrals of motion. Recall that in a rotating reference frame parallel to the principal axes of inertia of the rigid body, they read in general form as

$$
\dot{\vec{L}}+\vec{\omega} \times \vec{L}=\overrightarrow{0}
$$

where $\vec{L}=\boldsymbol{I} \vec{\omega}$ is the angular momentum, with $\boldsymbol{I}$ the matrix of moments of inertia and $\vec{\omega}$ the angular velocity about the principal axes. By setting $T:=\frac{1}{2} \vec{L} \cdot \vec{\omega}$ we can write the components of this equation in the principal reference frame, wherein $\boldsymbol{I}$ is constant, as the bi-Hamiltonian equations

$$
\dot{L}_{i}=\left\{L_{i}, \vec{L}^{2}, T\right\}
$$

for the Nambu bracket $\{f, g, h\}=\varepsilon^{i j k} \partial_{L_{i}} f \cdot \partial_{L_{j}} g \cdot \partial_{L_{k}} h$ on $\mathbb{R}^{3}$.

In the case of the $R$-flux background, the pertinent classical tribracket is given by

$$
\{f, g, h\}_{R}=-\frac{\ell_{s}^{4}}{\hbar^{2}} R^{i j k} \partial_{i} f \cdot \partial_{j} g \cdot \partial_{k} h .
$$

Our nonassociative phase space star product quantizes these tribrackets $[89,90]$, in the sense of the non-vanishing Jacobiators (5.13); this extends the quantization provided by the configuration space triproducts from (5.20). In particular, in the semi-classical limit one has $[f, g, h]_{\star}=-\hbar^{2}\{f, g, h\}_{R}+$ ... The issue of quantizing Nambu brackets is a longstanding problem, see e.g. [105, 106, 48, 49] for some approaches. Our phase space approach may assist by exploiting the various properties infered by the origin of the tribracket from the star product. For example, one of the formality conditions implies the pentagon identity [89]

$$
[f \star g, h, k]_{\star}-[f, g \star h, k]_{\star}+[f, g, h \star k]_{\star}=f \star[g, h, k]_{\star}+[f, g, h]_{\star} \star k
$$

for the Jacobiator, analogously to (5.16). This can be regarded as a 'quantum Leibniz rule', in the sense that at semiclassical order in the $R$-flux it coincides with the Leibniz rule for the classical 
tribracket (5.51). At present it is not clear what should serve as the quantum version of the classical fundamental identity, nor if one is really necessary, see e.g. [48] for a discussion of this point. The viability of our phase space model for the quantization of Nambu-Poisson structures is still to be thoroughly investigated.

\section{Further developments}

We conclude in this final section by briefly describing some extensions of the story described in this paper so far, all of which have important open problems which should be subject to future investigations.

\subsection{Nonassociative gravity}

One important omission from our considerations thus far has been addressing Question (Q3) from Section 1. A noncommutative theory of gravity on Moyal-Weyl spacetimes was constructed in [14], and is discussed in the lectures by L. Castellani at this School. As a fundamental length is incompatible with diffeomorphism symmetries, general relativity on noncommutative spacetime requires a Drinfeld twist via a two-cocycle of the Hopf enveloping algebra of the Lie algebra of vector fields (see e.g. [112] for a review). This defines a twisted tensor calculus, and leads to deformed Einstein equations [14]. Two major problems with this approach are that the Einstein equations are not generically real and so have questionable physical (and geometric) significance, and that twisted diffeomorphisms do not appear to be symmetries of string theory [4].

An analogous treatment can be followed to formulate a nonassociative theory of gravity, by defining a quasi-Hopf algebra of twisted diffeomorphisms with a two-cochain twist whose coboundary is the three-cocycle that controls nonassociativity, i.e. the associator $\varphi$, see (5.14). Such an approach was originally suggested by [90], and the pentagon relations (5.16) were used to consistently build nonassociative field theories in $[88,21]$. The cochain twisting method was extended by $[19,20]$ to develop a rigorous and very general theory of nonassociative differential geometry, extending and generalizing the considerations of [10] in the associative case, which in particular leads to a vielbein or first order formalism for nonassociative gravity. The metric aspects were considered in [27, 12], and currently nonassociative Riemannian geometry is under further construction.

The problems with noncommutative gravity alluded to above are of course still present in this theory (together with many additional issues). But recalling the development of our perspective, we need to explore how gravity on phase space induces gravity on configuration space. This problem was addressed by [11], where it is shown how the nonassociative geometry of phase space can lead to deformations of configuration space geometry. The idea is to remove momentum dependence up to an $O(d, d)$-transformation, which is the structure group symmetry determined by the natural phase space metric $\gamma=\mathrm{d} x^{i} \otimes \mathrm{d} p_{i}+\mathrm{d} p_{i} \otimes \mathrm{d} x^{i}$, analogously to double field theory. This is achieved by choosing a polarization, i.e. a maximally isotropic splitting $T\left(T^{*} M\right) \simeq L \oplus L^{*}$ of the tangent bundle of phase space with respect to the metric $\gamma$, and considering foliated tensor fields $T$ :

$$
\iota_{Z} T=0=£_{Z} T
$$


for every section $Z$ of the rank $d$ vector bundle $L^{*} \rightarrow T^{*} M$; if the distribution $L$ is integrable, then by Frobenius' theorem it can be (locally) identified with the tangent bundle of some $d$-dimensional submanifold of $T^{*} M$. For example, the foliation of phase space by leaves of constant momentum is determined by taking $L=T M$ and $Z_{i}=\frac{\partial}{\partial p_{i}}$ for $i=1, \ldots, d$, which formalizes the way in which we extracted the spacetime triproducts of fields occuring in closed string scattering amplitudes from the nonassociative phase space star product in (5.18).

The metric formulation of nonassociative gravity on phase space admits a Ricci tensor and a unique metric-compatible torsion-free connection, and in this way it yields a non-trivial deformation of the Ricci tensor of spacetime by locally non-geometric fluxes, which is given by [12]

$$
\begin{aligned}
\operatorname{Ric}_{i j}^{\circ}=\operatorname{Ric}_{i j}+\frac{\ell_{s}^{4}}{12} R^{a b c} & \left(\partial_{k}\left(\partial_{a} g^{k l}\left(\partial_{b} g_{l m}\right) \partial_{c} \Gamma_{i j}^{m}\right)-\partial_{j}\left(\partial_{a} g^{k l}\left(\partial_{b} g_{l m}\right) \partial_{c} \Gamma_{i k}^{m}\right)\right. \\
& +\partial_{c} g_{m n}\left(\partial_{a}\left(g^{l m} \Gamma_{l j}^{k}\right) \partial_{b} \Gamma_{i k}^{n}-\partial_{a}\left(g^{l m} \Gamma_{l k}^{k}\right) \partial_{b} \Gamma_{i j}^{n}\right. \\
& \left.\left.+\left(\Gamma_{i k}^{l} \partial_{a} g^{k m}-\partial_{a} \Gamma_{i k}^{l} g^{k m}\right) \partial_{b} \Gamma_{l j}^{n}-\left(\Gamma_{i j}^{l} \partial_{a} g^{k m}-\partial_{a} \Gamma_{i j}^{l} g^{k m}\right) \partial_{b} \Gamma_{l k}^{n}\right)\right)
\end{aligned}
$$

where $\mathrm{Ric}_{i j}$ is the usual Ricci tensor of the classical Levi-Civita connection $\Gamma_{i j}^{k}$ of a metric tensor $g_{i j}$ on spacetime. This expression is valid to linear order in the $R$-flux, which we recall is the order at which the corresponding conformal field theory calculations are reliable; this is also consistent with the second order $R$-flux corrections to the closed string equations (3.29) after T-duality, as the corrections (6.2) are indeed of second order $\ell_{s}^{4} \hbar$ in the double expansion in the parameters of the $R$-flux model. Notably, it is real, and thus represents the first non-trivial starting point for understanding how to define a nonassociative theory of gravity describing the low-energy effective dynamics of closed strings in non-geometric backgrounds. An action principle for nonassociative gravity is currently unknown, as some of the classical constructions of Riemannian geometry have yet to be generalized to the nonassociative setting.

The precise relation of this gravity theory with string theory and double field theory remains mysterious. Part of the issue is the role of twisted diffeomorphisms mentioned above. At any order in the string length scale $\ell_{s}^{2}$, the closed string effective action should be invariant under classical diffeomorphisms, while the effective action of double field theory should be invariant under generalized diffeomorphisms. Whereas the twisted diffeomorphism symmetries of phase space remain elusive, upon polarization to configuration space they may compare naturally with the expectations from string theory and double field theory. This was partially analysed by [11], but it is currently an open problem to understand precisely and systematically the meaning of the symmetries of nonassociative gravity on spacetime. In particular, one can ask if the effective theory retains the $O(d, d)$-symmetry of double field theory, in analogy to the case of open strings. Recall $[54,110]$ that noncommutative Yang-Mills theory on a $d$-dimensional torus is invariant under Morita equivalence, which is the structure inherited from T-duality in the decoupling limit. In this case the group $O(d, d)$ acts on the bivector $\theta$, the open string metric $G$, and the Yang-Mills coupling constant $g_{\mathrm{YM}}$ as

$$
\begin{aligned}
\theta & \longmapsto(A \theta+B)(C \theta+D)^{-1}, \\
G & \longmapsto(C \theta+D) G(C \theta+D)^{\top}, \\
g_{\mathrm{YM}} & \longmapsto g_{\mathrm{YM}}|\operatorname{det}(C \theta+D)|^{1 / 4},
\end{aligned}
$$


where $A, B, C, D$ are $d \times d$ matrices which parameterize an element of $O(d, d)$; this transformation is defined only on the subset of bivectors $\theta$ for which $C \theta+D$ is nondegenerate, which is dense for every element in the discrete subgroup $S O(d, d ; \mathbb{Z})$ of $O(d, d)$. It would be interesting to see if a similar set of T-duality transformation rules are global symmetries of nonassociative gravity.

\subsection{Higher structures in non-geometric M-theory}

It is natural to ask what becomes of the higher quantum geometry when we lift IIA string theory to M-theory, which is generally defined on a circle bundle

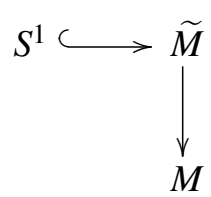

over the string target space $M$, with the string coupling $g_{s}$ realized geometrically as the radius $\lambda$ of the $S^{1}$ fibres. This has been discussed for lifts of the string theory $R$-flux model in three dimensions by [63], in the context of the $S L(5)$ exceptional field theory [25] which lifts the $O(3,3)$ double field theory. Taking the base space $M$ to be a three-dimensional twisted torus (the T-dual of the three-torus with $H$-flux), using the T-duality chain (3.1) we generate the string theory $R$-flux via a double T-duality transformation

$$
f_{j k}^{i} \stackrel{\mathscr{T}_{(j k)}}{\longrightarrow} R^{i j k}
$$

Let us lift this to M-theory on the trivial circle bundle $\widetilde{M}=M \times S^{1}$, with coordinates $x^{\mu}=\left(x^{i}, x^{4}\right)$ where $x^{4}$ is the local coordinate on $S^{1}$. Closed strings lift to closed M2-branes, and T-duality becomes U-duality which sends membrane wrapping modes $w^{i j}$ to momentum modes $p_{i}$. The Kalb-Ramond two-form field $B$ lifts to the three-form $C$-field of M-theory, and U-duality takes it to

$$
C_{\mu v \rho} \stackrel{\mathscr{U}_{(\mu \nu \rho)}}{\longrightarrow} \Omega^{\mu v \rho}
$$

where the trivector $\Omega^{\mu v \rho}$ defines the M-theory $R$-flux via its wrapping derivatives

$$
R^{\mu, v \rho \alpha \beta}=\partial^{\mu[v} \Omega^{\rho \alpha \beta]},
$$

which is the lift of the relation (3.19). The M-theory $R$-flux is a mixed symmetry tensor: It transforms as a vector in its first index and is antisymmetric in its last four indices. The particular choice $R^{4, \mu v \alpha \beta}=R \varepsilon^{\mu v \alpha \beta}$ breaks the $S L(5)$ symmetry to $S O(4)$.

The M2-brane phase space has a peculiar structure: The fact that there are no D0-branes on $M$ lifts to the statement that there are no momentum modes along the M-theory direction, i.e. $p_{4}=0$. In [63] it is conjectured that the covariant form of this constraint is given by

$$
R^{\mu, v \rho \alpha \beta} p_{\mu}=0
$$

and that the resulting seven-dimensional phase space has bracket structure given by

$$
\begin{array}{ll}
{\left[x^{i}, x^{j}\right]=\frac{\mathrm{i} \ell_{s}^{4}}{3 \hbar} R^{4, i j k 4} p_{k} \quad \text { and }} & {\left[x^{4}, x^{i}\right]=\frac{\mathrm{i} \lambda \ell_{s}^{4}}{3 \hbar} R^{4,1234} p^{i}} \\
{\left[x^{i}, p_{j}\right]=\mathrm{i} \hbar \delta_{j}^{i} x^{4}+\mathrm{i} \hbar \lambda \varepsilon^{i}{ }_{j k} x^{k}} & \text { and } \quad\left[x^{4}, p_{i}\right]=\mathrm{i} \hbar \lambda^{2} x_{i} \\
{\left[p_{i}, p_{j}\right]=-\mathrm{i} \hbar \lambda \varepsilon_{i j k} p^{k},} &
\end{array}
$$


with the Jacobiators

$$
\begin{aligned}
& {\left[x^{i}, x^{j}, x^{k}\right]=\frac{\mathrm{i} \ell_{s}^{4}}{3 \hbar} R^{4, i j k 4} x^{4} \quad \text { and } \quad\left[x^{i}, x^{j}, x^{4}\right]=-\frac{\mathrm{i} \lambda^{2} \ell_{s}^{4}}{3 \hbar} R^{4, i j k 4} x_{k},} \\
& {\left[p_{i}, x^{j}, x^{k}\right]=\frac{\mathrm{i} \lambda \ell_{s}^{4}}{3 \hbar} R^{4,1234}\left(\delta_{i}^{j} p^{k}-\delta_{i}^{k} p^{j}\right) \quad \text { and } \quad\left[p^{i}, x^{j}, x^{4}\right]=\frac{\mathrm{i} \lambda^{2} \ell_{s}^{4}}{3 \hbar} R^{4, i j k 4} p_{k},} \\
& {\left[p_{i}, p_{j}, x^{k}\right]=-\mathrm{i} \hbar \lambda^{2} \varepsilon_{i j}{ }^{k} x^{4}-\mathrm{i} \hbar \lambda\left(\delta_{j}{ }^{k} x_{i}-\delta_{i}{ }^{k} x_{j}\right) \quad \text { and } \quad\left[p_{i}, p_{j}, x^{4}\right]=\mathrm{i} \hbar \lambda^{3} \varepsilon_{i j k} x^{k},} \\
& {\left[p_{i}, p_{j}, p_{k}\right]=0 .}
\end{aligned}
$$

In the contraction limit $\lambda=0$, which is precisely the limit of weak string coupling $g_{s} \rightarrow 0$ sending M-theory to IIA string theory, these brackets reduce to those of the closed string $R$-flux algebra (3.27); in this case the M-theory direction $x^{4}$ becomes a central element of the contracted algebra, so we may set it to $x^{4}=1$.

These brackets originate from the nonassociative alternative algebra of octonions $\mathbb{O}$ by a suitable rescaling of the seven imaginary unit octonions [63]. Deformation quantization of this quasiPoisson structure was carried out in [81]. It proceeds via a choice of a $G_{2}$-structure, i.e. a cross product on the real inner product space $\mathbb{R}^{7}$ given by structure constants of $\mathbb{O}$ in a suitable oriented basis, which is preserved by rotations of $\mathbb{R}^{7}$ in the subgroup $G_{2} \subset S O(7)$. Using alternativity one can define octonion exponentials, and the corresponding nonassociative Baker-Campbell-Hausdorf formula is captured by a 2-group addition law on the seven-dimensional Fourier space, which gives the deformation quantization of the M2-brane phase space in terms of an explicit, albeit complicated, nonassociative phase space star product $f \star_{\lambda} g$. This quantization has a variety of interesting features which are described in [81]. For example, the corresponding configuration space triproducts $\Delta_{\lambda}$ defined by $\star_{\lambda}$ analogously to (5.18) quantize the 3-Lie algebra $A_{4}$ :

$$
\left[x^{\mu}, x^{v}, x^{\alpha}\right]_{\Delta_{1}}=\ell_{s}^{4} R \varepsilon^{\mu v \alpha \beta} x^{\beta}
$$

which is known from studies of multiple membranes in M-theory, see e.g. [15] for a review, and whose quantization has been previously largely unknown, see e.g. [48] for an earlier analysis of this problem. Moreover, the noncommutative M2-brane momentum space is familiar from the noncommutative spacetimes arising in three-dimensional quantum gravity, see e.g. [57]. Using $x^{4} \star_{\lambda} f=x^{4} f+O(\lambda)$ one finds that the M2-brane star product reduces non-trivially to the closed string star product (5.8) in the weak string coupling limit:

$$
\lim _{\lambda \rightarrow 0}(f \star \lambda g)\left(x^{i}, x^{4}\right)=(f \star g)\left(x^{i}\right) .
$$

In [81] it was also suggested how to extend the nonassociative M-theory $R$-flux algebra to the full unconstrained eight-dimensional phase space by a choice of a $\operatorname{Spin}(7)$-structure, i.e. a triple cross product on the real inner product space $\mathbb{R}^{8}$, which is parameterized by a four-form $\phi$ invariant under rotations of $\mathbb{R}^{8}$ in the subgroup $\operatorname{Spin}(7) \subset S O(8)$. This extends the representation of the cross product to all real and imaginary octonions, and it defines the eight-dimensional "covariant" M2- 
brane phase space 3-algebra with the $S O(4) \times S O(4)$-symmetric 3-brackets given by

$$
\begin{aligned}
& {\left[x^{i}, x^{j}, x^{k}\right]_{\phi}=-\frac{\ell_{s}^{4}}{2} R^{4, i j k 4} x^{4} \quad \text { and } \quad\left[x^{i}, x^{j}, x^{4}\right]_{\phi}=\frac{\lambda^{2} \ell_{s}^{4}}{2} R^{4, i j k 4} x_{k},} \\
& {\left[p^{i}, x^{j}, x^{k}\right]_{\phi}=-\frac{\lambda^{2} \ell_{s}^{4}}{2} R^{4, i j k 4} p_{4}-\frac{\lambda \ell_{s}^{4}}{2} R^{4, i j k l} p_{l},} \\
& {\left[p^{i}, x^{j}, x^{4}\right]_{\phi}=-\frac{\lambda^{2} \ell_{s}^{4}}{2} R^{4,1234} \delta^{i j} p_{4}-\frac{\lambda^{2} \ell_{s}^{4}}{2} R^{4, i j k 4} p_{k},} \\
& {\left[p_{i}, p_{j}, x^{k}\right]_{\phi}=\frac{\lambda^{2}}{2} \varepsilon_{i j}{ }^{k} x^{4}+\frac{\hbar^{2} \lambda}{2}\left(\delta_{j}{ }^{k} x_{i}-\delta_{i}^{k} x_{j}\right),} \\
& {\left[p_{i}, p_{j}, x^{4}\right]_{\phi}=-\frac{\hbar^{2} \lambda^{3}}{2} \varepsilon_{i j k} x^{k} \quad \text { and } \quad\left[p_{i}, p_{j}, p_{k}\right]_{\phi}=-2 \hbar^{2} \lambda \varepsilon_{i j k} p_{4},} \\
& {\left[p_{4}, x^{i}, x^{j}\right]_{\phi}=\frac{\lambda \ell_{s}^{4}}{2} R^{4, i j k 4} p_{k} \quad \text { and } \quad\left[p_{4}, x^{i}, x^{4}\right]_{\phi}=-\frac{\lambda^{2} \ell_{s}^{4}}{2} R^{4,1234} p^{i},} \\
& {\left[p_{4}, p_{i}, x^{j}\right]_{\phi}=-\frac{\hbar^{2} \lambda}{2} \delta_{i}^{j} x^{4}-\frac{\hbar^{2} \lambda^{2}}{2} \varepsilon_{i}{ }^{j k} x_{k},} \\
& {\left[p_{4}, p_{i}, x^{4}\right]_{\phi}=-\frac{\hbar^{2} \lambda^{3}}{2} x_{i} \quad \text { and } \quad\left[p_{4}, p_{i}, p_{j}\right]_{\phi}=-\frac{\hbar^{2} \lambda^{2}}{2} \varepsilon_{i j k} p^{k} .}
\end{aligned}
$$

For any constraint $G=0$ on the eight-dimensional phase space, these 3-brackets induce a 2-bracket by defining $[f, g]_{G}:=[f, g, G]_{\phi}$. In particular, taking $G=\frac{2}{\lambda \hbar^{2}} p_{4}$ (or $G=R^{\mu, v \rho \alpha \beta} p_{\mu}$ ) gauge fixes the 3-brackets (6.13) to the brackets (6.9) together with their Jacobiators. The quantization of this 3algebra is currently an open problem; see [81] for some preliminary steps towards the construction of a suitable ternary product for deformation quantization which naturally incorporates both the star product and the triproduct.

The magnetic monopole system of Section 2 with constant uniform magnetic charge can be embedded in string theory as D0-branes bound to a uniform distribution of D6-branes. The Mtheory lift of this configuration is identified by [87] as a non-geometric variant of the Kaluza-Klein monopole solution of M-theory, whose phase space brackets map to (6.9) under magnetic duality and reduce to the magnetic monopole algebra (2.3) in the contraction limit $\lambda=0$.

A suitable check of these purported claims should come from the next rung $n=3$ on the AKSZ geometric ladder of Section 4, which has been investigated partially in [79]; the symplectic Lie 3algebroid structure in this case is called a Lie algebroid up to homotopy, and the corresponding AKSZ sigma-model describes closed M2-branes viewed as boundaries of open threebranes. The standard Lie algebroid up to homotopy is the anticipated generalized tangent bundle $T \widetilde{M} \oplus \bigwedge^{2} T^{*} \widetilde{M}$ of exceptional field theory [22], and the bracket structure in this case is the expected higher 2Courant bracket. However, beyond the simplest case of the four-form flux $G=\mathrm{d} C$ of the M-theory $C$-field, it is not clear how to twist these higher algebroid structures by geometric and non-geometric fluxes; see [79] for a discussion of this point. This may be related to the fact that, unlike T-duality which maps closed strings to closed strings, U-duality maps M2-branes to M-waves (the lifts of D0-branes), and more generally to M5-branes in higher dimensions.

Locally non-geometric fluxes in M-theory on higher dimensional spacetimes have been discussed in [86]. In each dimensionality one encounters the same qualitative structure, missing mo- 
mentum modes in the M2-brane phase space, but now with a host of $R$-type fluxes with varying tensorial structures. The corresponding bracket structures are not currently understood, and may also involve higher algebras.

\section{3 $L_{\infty}$-algebras}

As mentioned in Section 5, the Kontsevich formality maps are quasi-isomorphisms between particular examples of $L_{\infty}$-algebras, and it is natural to wonder if such higher algebraic structures govern the infinitesimal symmetries of the higher quantum geometries we have discussed. In [89] it was observed that the nonassociative phase space deformation (3.27) underlying the closed string $R$-flux background can be realized and understood as a 2-term $L_{\infty}$-algebra, which is similar to the characterization [97] of the bracket structure underlying Courant algebroids from Section 4. This result was extended, and put into a more general and systematic framework by [70], showing in particular how the M-theory $R$-flux algebra (6.9) is realized in the same way. It would be interesting to similarly understand the covariant M2-brane 3-algebra (6.13) in this way, which may also be the appropriate framework for the higher bracket structures underlying the phase spaces of nongeometric M-theory in dimensions $d>4$ where they are governed by the $E_{d}$ exceptional field theory [86].

These occurences nicely match the original appearence of $L_{\infty}$-algebras in physics as higher gauge symmetry algebras of closed string field theory in [119], and more recently as the higher symmetries underlying double field theory $[51,50,68]$ and two-dimensional conformal field theory [29]. It was shown by [30] that the symmetries and dynamics of open string nonassociative gauge theories on curved D-brane worldvolumes are also governed by an underlying $L_{\infty}$-algebra. Extrapolating this feature to non-geometric backgrounds could lead to a similar characterization of the diffeomorphism symmetries in nonassociative gravity on phase space, and its fate under polarization to spacetime which may clarify the connections with closed string theory and double field theory discussed above.

\section{Acknowledgments}

We would like to thank the organisors of the School for the invitation to deliver these lectures, and all the participants for their persistent questions and comments which have helped shape the structure and content of these lecture notes. Several points of view discussed in this article have emerged from numerous discussions over the years with various collaborators and colleagues; we would like to thank in particular Paolo Aschieri, Ralph Blumenhagen, Chris Hull, Dieter Lüst, Vladislav Kupriyanov, Emanuel Malek, Alexander Schenkel and Peter Schupp. This work was supported in part by the Action MP1405 QSPACE from the European Cooperation in Science and Technology (COST) and by the Consolidated Grant ST/P000363/1 from the UK Science and Technology Facilities Council (STFC).

\section{References}

[1] G. Aldazabal, D. Marqués and C. Núñez, "Double field theory: A pedagogical review," Class. Quant. Grav. 30, 163001 (2013) [arXiv:1305.1907 [hep-th]]. 
[2] A. Alekseev and T. Strobl, "Current algebras and differential geometry," JHEP 0503, 035 (2005) [arXiv:hep-th/0410183].

[3] M. Alexandrov, M. Kontsevich, A. Schwarz and O. Zaboronsky, "The geometry of the master equation and topological quantum field theory,” Int. J. Mod. Phys. A 12, 1405-1429 (1997) [arXiv:hep-th/9502010].

[4] L. Álvarez-Gaumé, F. Meyer and M.A. Vázquez-Mozo, "Comments on noncommutative gravity," Nucl. Phys. B 753 (2006) 92-127 [arXiv:hep-th/0605113].

[5] D. Andriot, M. Larfors, D. Lüst and P. Patalong, "(Non)commutative closed string on T-dual toroidal backgrounds,” JHEP 1306, 021 (2013) [arXiv:1211.6437 [hep-th]].

[6] D. Andriot, O. Hohm, M. Larfors, D. Lüst and P. Patalong, "A geometric action for non-geometric fluxes,” Phys. Rev. Lett. 108, 261602 (2012) [arXiv:1202.3060 [hep-th]].

[7] D. Andriot, O. Hohm, M. Larfors, D. Lüst and P. Patalong, "Non-geometric fluxes in supergravity and double field theory," Fortsch. Phys. 60, 1150-1186 (2012) [arXiv:1204.1979 [hep-th]].

[8] F. Ardalan, H. Arfaei and M.M. Sheikh-Jabbari, "Mixed branes and M(atrix) theory on noncommutative torus," in: PASCOS 1998 (World Scientific, Singapore), 653-656 (1999) [arXiv:hep-th/9803067].

[9] F. Ardalan, H. Arfaei and M.M. Sheikh-Jabbari, "Noncommutative geometry from strings and branes," JHEP 9902, 016 (1999) [arXiv:hep-th/9810072].

[10] P. Aschieri and A. Schenkel, "Noncommutative connections on bimodules and Drinfeld twist deformation,” Adv. Theor. Math. Phys. 18, 513-612 (2014) [arXiv:1210.0241 [math.QA]].

[11] P. Aschieri and R.J. Szabo, "Triproducts, nonassociative star products and geometry of $R$-flux string compactifications,” J. Phys. Conf. Ser. 634, 012004 (2015) [arXiv:1504.03915 [hep-th]].

[12] P. Aschieri, M. Dimitrijević Ćirić and R.J. Szabo, "Nonassociative differential geometry and gravity with non-geometric fluxes,” JHEP 1802, 036 (2018) [arXiv:1710.11467 [hep-th]].

[13] P. Aschieri, M. Dimitrijević, F. Meyer and J. Wess, "Noncommutative geometry and gravity," Class. Quant. Grav. 23, 1883-1912 (2006) [arXiv:hep-th/0510059].

[14] P. Aschieri, C. Blohmann, M. Dimitrijević, F. Meyer, P. Schupp and J. Wess, "A gravity theory on noncommutative spaces," Class. Quant. Grav. 22, 3511-3532 (2005) [arXiv:hep-th/0504183].

[15] J. Bagger, N. Lambert, S. Mukhi and C. Papageorgakis, "Multiple membranes in M-theory," Phys. Rept. 527, 1-100 (2013) [arXiv:1203.3546 [hep-th]].

[16] I. Bakas and D. Lüst, "Three-cocycles, nonassociative star products and the magnetic paradigm of $R$-flux string vacua,” JHEP 1401, 171 (2014) [arXiv:1309.3172 [hep-th]].

[17] I. Bakas and D. Lüst, "T-duality, quotients and currents for non-geometric closed strings," Fortsch. Phys. 63, 543-570 (2015) [arXiv:1505.04004 [hep-th]].

[18] I. Bakhmatov, E.Ó. Colgáin, M.M. Sheikh-Jabbari and H. Yavartanoo, "Yang-Baxter deformations beyond coset spaces (a slick way to do TsT)," arXiv:1803.07498 [hep-th].

[19] G.E. Barnes, A. Schenkel and R.J. Szabo, "Nonassociative geometry in quasi-Hopf representation categories I: Bimodules and their internal homomorphisms,” J. Geom. Phys. 89, 111-152 (2015) [arXiv:1409.6331 [math.QA]]. 
[20] G.E. Barnes, A. Schenkel and R.J. Szabo, "Nonassociative geometry in quasi-Hopf representation categories II: Connections and curvature,” J. Geom. Phys. 106, 234-255 (2016) [arXiv:1507.02792 [math.QA]].

[21] G.E. Barnes, A. Schenkel and R.J. Szabo, "Working with nonassociative geometry and field theory," PoS CORFU 2015, 081 (2016) [arXiv:1601.07353 [hep-th]].

[22] D.S. Berman and D.C. Thompson, "Duality symmetric string and M-theory," Phys. Rept. 566, 1-60 (2014) [arXiv:1306.2643 [hep-th]].

[23] T. Bessho, M.A. Heller, N. Ikeda and S. Watamura, "Topological membranes, current algebras and $H$-flux- $R$-flux duality based on Courant algebroids,” JHEP 1604, 170 (2016) [arXiv:1511.03425 [hep-th]].

[24] C.D.A. Blair, "Noncommutativity and nonassociativity of the doubled string in non-geometric backgrounds," JHEP 1506, 091 (2015) [arXiv:1405.2283 [hep-th]].

[25] C.D.A. Blair and E. Malek, "Geometry and fluxes of $S L(5)$ exceptional field theory," JHEP 1503, 144 (2015) [arXiv:1412.0635 [hep-th]].

[26] R. Blumenhagen, "A course on noncommutative geometry in string theory," Fortsch. Phys. 62, 709-726 (2014) [arXiv:1403.4805 [hep-th]].

[27] R. Blumenhagen and M. Fuchs, “Towards a theory of nonassociative gravity,” JHEP 1607, 019 (2016) [arXiv:1604.03253 [hep-th]].

[28] R. Blumenhagen and E. Plauschinn, “Nonassociative gravity in string theory?,” J. Phys. A 44, 015401 (2011) [arXiv:1010.1263 [hep-th]].

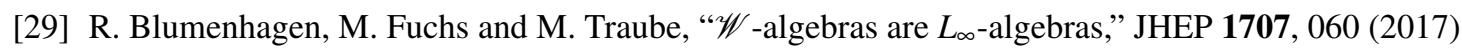
[arXiv:1705.00736 [hep-th]].

[30] R. Blumenhagen, I. Brunner, V.G. Kupriyanov and D. Lüst, "Bootstrapping noncommutative gauge theories from $L_{\infty}$-algebras," arXiv:1803.00732 [hep-th].

[31] R. Blumenhagen, A. Deser, E. Plauschinn and F. Rennecke, "Bianchi identities for non-geometric fluxes: From quasi-Poisson structures to Courant algebroids," Fortsch. Phys. 60, 1217-1228 (2012) [arXiv:1205.1522 [hep-th]].

[32] R. Blumenhagen, B. Körs, D. Lüst and S. Stieberger, "Four-dimensional string compactifications with D-branes, orientifolds and fluxes," Phys. Rept. 445, 1-193 (2007) [arXiv:hep-th/0610327].

[33] R. Blumenhagen, A. Deser, D. Lüst, E. Plauschinn and F. Rennecke, "Non-geometric fluxes, asymmetric strings and nonassociative geometry,” J. Phys. A 44, 385401 (2011) [arXiv:1106.0316 [hep-th]].

[34] R. Blumenhagen, M. Fuchs, F. Haßler, D. Lüst and R. Sun, "Nonassociative deformations of geometry in double field theory," JHEP 1404, 141 (2014) [arXiv:1312.0719 [hep-th]].

[35] M. Bojowald, S. Brahma and U. Büyükçam, “Testing nonassociative quantum mechanics,” Phys. Rev. Lett. 115, 220402 (2015) [Erratum: Phys. Rev. Lett. 117, 099901 (2016)] [arXiv:1510.07559 [quant-ph]].

[36] M. Bojowald, S. Brahma, U. Büyükçam and T. Strobl, "States in nonassociative quantum mechanics: Uncertainty relations and semiclassical evolution,” JHEP 1503, 093 (2015) [arXiv:1411.3710 [hep-th]]. 
[37] M. Bojowald, S. Brahma, U. Büyükçam and T. Strobl, "Monopole star products are non-alternative," JHEP 1704, 028 (2017) [arXiv:1610.08359 [math-ph]].

[38] P. Bouwknegt, "Lectures on cohomology, T-duality, and generalized geometry," Lect. Notes Phys. 807, 261-311 (2010).

[39] S. Bunk, L. Müller and R.J. Szabo, "Geometry and 2-Hilbert space for nonassociative magnetic translations," in preparation.

[40] S. Bunk, C. Saemann and R.J. Szabo, “The 2-Hilbert space of a prequantum bundle gerbe,” Rev. Math. Phys. 30, 1850001 (2017) [arXiv:1608.08455 [math-ph]].

[41] A.S. Cattaneo and G. Felder, "A path integral approach to the Kontsevich quantization formula," Commun. Math. Phys. 212, 591-611 (2000) [arXiv:math.QA/9902090].

[42] A. Chatzistavrakidis and L. Jonke, "Matrix theory origins of non-geometric fluxes," JHEP 1302, 040 (2013) [arXiv:1207.6412 [hep-th]].

[43] A. Chatzistavrakidis, L. Jonke and O. Lechtenfeld, "Sigma-models for genuinely non-geometric backgrounds,” JHEP 1511, 182 (2015) [arXiv:1505.05457 [hep-th]].

[44] A. Chatzistavrakidis, L. Jonke, F.S. Khoo and R.J. Szabo, "Double field theory and membrane sigma-models," arXiv:1802.07003 [hep-th].

[45] C.-S. Chu and P.-M. Ho, "Noncommutative open string and D-brane," Nucl. Phys. B 550, 151-168 (1999) [arXiv:hep-th/9812219].

[46] C. Condeescu, I. Florakis and D. Lüst, "Asymmetric orbifolds, non-geometric fluxes and noncommutativity in closed string theory," JHEP 1204, 121 (2012) [arXiv:1202.6366 [hep-th]].

[47] L. Davidović, B. Nikolić and B. Sazdović, "Canonical approach to the closed string noncommutativity,” Eur. Phys. J. C 74, 2734 (2014) [arXiv:1307.6158 [hep-th]].

[48] J. DeBellis, C. Saemann and R.J. Szabo, "Quantized Nambu-Poisson manifolds and n-Lie algebras," J. Math. Phys. 51, 122303 (2010) [arXiv:1001.3275 [hep-th]].

[49] J. DeBellis, C. Saemann and R.J. Szabo, "Quantized Nambu-Poisson manifolds in a 3-Lie algebra reduced model,” JHEP 1104, 075 (2011) [arXiv:1012.2236 [hep-th]].

[50] A. Deser and C. Saemann, "Extended Riemannian geometry I: Local double field theory," arXiv:1611.02772 [hep-th].

[51] A. Deser and J. Stasheff, "Even symplectic supermanifolds and double field theory," Commun. Math. Phys. 339, 1003-1020 (2015) [arXiv:1406.3601 [math-ph]].

[52] M.R. Douglas and C.M. Hull, “D-branes and the noncommutative torus,” JHEP 9802, 008 (1998) [arXiv:hep-th/9711165].

[53] M.R. Douglas and S. Kachru, "Flux compactification,” Rev. Mod. Phys. 79, 733-796 (2007) [arXiv:hep-th/0610102].

[54] M.R. Douglas and N.A. Nekrasov, "Noncommutative field theory," Rev. Mod. Phys. 73, 977-1029 (2001) [arXiv:hep-th/0106048].

[55] M.J. Duff, "Duality rotations in string theory,” Nucl. Phys. B 335, 610-620 (1990).

[56] D.S. Freed and E. Witten, “Anomalies in string theory with D-branes," Asian J. Math. 3, 819-852 (1999) [arXiv:hep-th/9907189]. 
[57] L. Freidel and E.R. Livine, "Effective $3 D$ quantum gravity and noncommutative quantum field theory," Phys. Rev. Lett. 96 (2006) 221301 [arXiv:hep-th/0512113].

[58] L. Freidel, R.G. Leigh and D. Minic, “Metastring theory and modular spacetime," JHEP 1506, 006 (2015) [arXiv:1502.08005 [hep-th]].

[59] L. Freidel, F.J. Rudolph and D. Svoboda, “Generalized kinematics for double field theory," JHEP 1711, 175 (2017) [arXiv:1706.07089 [hep-th]].

[60] M. Graña, "Flux compactifications in string theory: A comprehensive review," Phys. Rept. 423, 91-158 (2006) [arXiv:hep-th/0509003].

[61] M. Gualtieri, "Generalized complex geometry," PhD Thesis, University of Oxford [arXiv:math-dg/0401221].

[62] M. Günaydin and B. Zumino, "Magnetic charge and nonassociative algebras," in: Old and New Problems in Fundamental Physics: Meeting in Honour of G.C. Wick (Quaderni, Pisa: Scuola Normale Superiore), 43-53 (1986).

[63] M. Günaydin, D. Lüst and E. Malek, "Nonassociativity in non-geometric string and M-theory backgrounds, the algebra of octonions, and missing momentum modes," JHEP 1611, 027 (2016) [arXiv:1607.06474 [hep-th]].

[64] N. Halmagyi, "Non-geometric string backgrounds and worldsheet algebras,” JHEP 0807, 137 (2008) [arXiv:0805.4571 [hep-th]].

[65] N. Halmagyi, "Non-geometric backgrounds and the first order string sigma-model,” arXiv:0906.2891 [hep-th].

[66] N. Hitchin, “Generalized Calabi-Yau manifolds,” Quart. J. Math. 54, 281-308 (2003) [arXiv:math-dg/0209099].

[67] P.-M. Ho and Y. Matsuo, “The Nambu bracket and M-theory,” PTEP 2016, 06A104 (2016) [arXiv:1603.09534 [hep-th]].

[68] O. Hohm and B. Zwiebach, “ $L_{\infty}$-algebras and field theory,” Fortsch. Phys. 65, 1700014 (2017) [arXiv:1701.08824 [hep-th]].

[69] O. Hohm, D. Lüst and B. Zwiebach, "The spacetime of double field theory: Review, remarks, and outlook,” Fortsch. Phys. 61, 926-966 (2013) [arXiv:1309.2977 [hep-th]].

[70] O. Hohm, V.G. Kupriyanov, D. Lüst and M. Traube, "General constructions of $L_{\infty}$-algebras," arXiv:1709.10004 [math-ph].

[71] C.M. Hull, “A geometry for non-geometric string backgrounds,” JHEP 0510, 065 (2005) [arXiv:hep-th/0406102].

[72] C.M. Hull and R.A. Reid-Edwards, "Non-geometric backgrounds, doubled geometry and generalized T-duality,” JHEP 0909, 014 (2009) [arXiv:0902.4032 [hep-th]].

[73] C.M. Hull and B. Zwiebach, "Double field theory,” JHEP 0909, 099 (2009) [arXiv:0904.4664 [hep-th]].

[74] N. Ikeda, “Two-dimensional gravity and nonlinear gauge theory,” Ann. Phys. 235, 435-464 (1994) [arXiv:hep-th/9312059].

[75] N. Ikeda, "Lectures on AKSZ sigma-models for physicists," in: Noncommutative Geometry and Physics 4, eds. Y. Maeda, H. Moriyoshi, M. Kotani and S. Watamura (World Scientific), 79-170 (2017) [arXiv:1204.3714 [hep-th]]. 
[76] R. Jackiw, “Three-cocycle in mathematics and physics,” Phys. Rev. Lett. 54, 159-162 (1985).

[77] R. Jackiw, “Dirac's magnetic monopoles (again),” Int. J. Mod. Phys. A 19S1, 137-144 (2004) [arXiv:hep-th/0212058].

[78] C. Klimčík and T. Strobl, “WZW-Poisson manifolds,” J. Geom. Phys. 43, 341-344 (2002) [arXiv:math.SG/0104189].

[79] Z. Kökényesi, A. Sinkovics and R.J. Szabo, "AKSZ constructions for topological membranes on $G_{2}$-manifolds," arXiv:1802.04581 [hep-th].

[80] M. Kontsevich, "Deformation quantization of Poisson manifolds," Lett. Math. Phys. 66, 157-216 (2003) [arXiv:q-alg/9709040].

[81] V.G. Kupriyanov and R.J. Szabo, " $G_{2}$-structures and quantization of non-geometric M-theory backgrounds,” JHEP 1702, 099 (2017) [arXiv:1701.02574 [hep-th]].

[82] V.G. Kupriyanov and R.J. Szabo, "Symplectic realisation of electric charge in fields of monopole distributions," arXiv:1803.00405 [hep-th].

[83] V.G. Kupriyanov and D.V. Vassilevich, “Nonassociative Weyl star products,” JHEP 1509, 103 (2015) [arXiv:1506.02329 [hep-th]].

[84] D. Lüst, “T-duality and closed string noncommutative (doubled) geometry,” JHEP 1012, 084 (2010) [arXiv:1010.1361 [hep-th]].

[85] D. Lüst, "Twisted Poisson structures and noncommutative/nonassociative closed string geometry," PoS CORFU 2011, 086 (2011) [arXiv:1205.0100 [hep-th]].

[86] D. Lüst, E. Malek and M. Syväri, "Locally non-geometric fluxes and missing momenta in M-theory," JHEP 1801, 050 (2018) [arXiv:1710.05919 [hep-th]].

[87] D. Lüst, E. Malek and R.J. Szabo, "Non-geometric Kaluza-Klein monopoles and magnetic duals of M-theory R-flux backgrounds," JHEP 1710 (2017) 144 [arXiv:1705.09639 [hep-th]].

[88] D. Mylonas and R.J. Szabo, "Nonassociative field theory on non-geometric spaces," Fortsch. Phys. 62, 727-732 (2014) [arXiv:1404.7304 [hep-th]].

[89] D. Mylonas, P. Schupp and R.J. Szabo, "Membrane sigma-models and quantization of non-geometric flux backgrounds,” JHEP 1209, 012 (2012) [arXiv:1207.0926 [hep-th]].

[90] D. Mylonas, P. Schupp and R.J. Szabo, "Non-geometric fluxes, quasi-Hopf twist deformations and nonassociative quantum mechanics,” J. Math. Phys. 55, 122301 (2014) [arXiv:1312.1621 [hep-th]].

[91] D. Mylonas, P. Schupp and R.J. Szabo, "Nonassociative geometry and twist deformations in non-geometric string theory,” PoS ICMP 2013, 007 (2013) [arXiv:1402.7306 [hep-th]].

[92] Y. Nambu, “Generalized Hamiltonian dynamics,” Phys. Rev. D 7, 2405-2412 (1973).

[93] B. Nikolić and D. Obrić, "Noncommutativity and nonassociativity of closed bosonic string on T-dual toroidal backgrounds," arXiv:1801.08772 [hep-th].

[94] E. Plauschinn, "Non-geometric fluxes and nonassociative geometry," PoS CORFU 2011, 061 (2011) [arXiv:1203.6203 [hep-th]].

[95] D. Roytenberg, "On the structure of graded symplectic supermanifolds and Courant algebroids," Contemp. Math. 315, 169-186 (2002) [arXiv:math.SG/0203110].

[96] D. Roytenberg, "AKSZ-BV formalism and Courant algebroid-induced topological field theories," Lett. Math. Phys. 79, 143-159 (2007) [arXiv:hep-th/0608150]. 
[97] D. Roytenberg and A. Weinstein, "Courant algebroids and strongly homotopy Lie algebras," Lett. Math. Phys. 46, 81-93 (1998) [arXiv:math.QA/9802118].

[98] C. Saemann and R.J. Szabo, “Groupoid quantization of loop spaces,” PoS CORFU 2011, 046 (2011) [arXiv:1203.5921 [hep-th]].

[99] C. Saemann and R.J. Szabo, "Groupoids, loop spaces and quantization of 2-plectic manifolds," Rev. Math. Phys. 25, 1330005 (2013) [arXiv:1211.0395 [hep-th]].

[100] P. Schaller and T. Strobl, "Poisson structure induced (topological) field theories," Mod. Phys. Lett. A 9, 3129-3136 (1994) [arXiv:hep-th/9405110].

[101] V. Schomerus, “D-branes and deformation quantization,” JHEP 9906, 030 (1999) [arXiv:hep-th/9903205].

[102] N. Seiberg and E. Witten, "String theory and noncommutative geometry," JHEP 9909, 032 (1999) [arXiv:hep-th/9908142].

[103] M.M. Sheikh-Jabbari, "Super Yang-Mills theory on noncommutative torus from open strings interactions," Phys. Lett. B 450, 119-125 (1999) [arXiv:hep-th/9810179].

[104] M.M. Sheikh-Jabbari, "Open strings in a B-field background as electric dipoles,” Phys. Lett. B 455 , 129-134 (1999) [arXiv:hep-th/9901080].

[105] M.M. Sheikh-Jabbari, "Tiny graviton matrix theory: DLCQ of IIB plane-wave string theory, a conjecture,” JHEP 0409, 017 (2004) [arXiv:hep-th/0406214].

[106] M.M. Sheikh-Jabbari and M. Torabian, "Classification of all $\frac{1}{2}$ BPS solutions of the tiny graviton matrix theory," JHEP 0504, 001 (2005) [arXiv:hep-th/0501001].

[107] J. Shelton, W. Taylor and B. Wecht, "Non-geometric flux compactifications," JHEP 0510, 085 (2005) [arXiv:hep-th/0508133].

[108] W. Siegel, “Two vierbein formalism for string inspired axionic gravity," Phys. Rev. D 47, 5453-5459 (1993) [arXiv:hep-th/9302036].

[109] W. Siegel, “Superspace duality in low-energy superstrings,” Phys. Rev. D 48, 2826-2837 (1993) [arXiv:hep-th/9305073].

[110] R.J. Szabo, “Quantum field theory on noncommutative spaces,” Phys. Rept. 378, 207-299 (2003) [arXiv:hep-th/0109162].

[111] R.J. Szabo, “Magnetic backgrounds and noncommutative field theory,” Int. J. Mod. Phys. A 19, 1837-1862 (2004) [arXiv:physics/0401142].

[112] R.J. Szabo, "Symmetry, gravity and noncommutativity," Class. Quant. Grav. 23, R199-R242 (2006) [arXiv:hep-th/0606233].

[113] R.J. Szabo, "Quantum gravity, field theory and signatures of noncommutative spacetime," Gen. Rel. Grav. 42, 1-29 (2010) [arXiv:0906.2913 [hep-th]].

[114] R.J. Szabo, "Magnetic monopoles and nonassociative deformations of quantum theory," J. Phys. Conf. Ser. 965, 012041 (2018) [arXiv:1709.10080 [hep-th]].

[115] L. Takhtajan, “On foundation of the generalized Nambu mechanics,” Commun. Math. Phys. 160, 295-316 (1994) [arXiv:hep-th/9301111].

[116] D.V. Vassilevich and F.M.C. Oliveira, "Nearly associative deformation quantization," arXiv:1802.05808 [math-ph]. 
[117] B. Wecht, "Lectures on non-geometric flux compactifications," Class. Quant. Grav. 24, S773-S794 (2007) [arXiv:0708.3984 [hep-th]].

[118] C.K. Zachos, "Deformation quantization: Quantum mechanics lives and works in phase space," Int. J. Mod. Phys. A 17, 297-316 (2002) [arXiv:hep-th/0110114].

[119] B. Zwiebach, "Closed string field theory: Quantum action and the BV master equation," Nucl. Phys. B 390, 33-152 (1993) [arXiv:hep-th/9206084]. 